\title{
Operational Modal Identification using Variational Bayes
}

\author{
Binbin Li \\ $\mathrm{PhD}$ Candidate, Civil and Environmental Engineering, \\ University of California, Berkeley, CA 94720. Phone: (510) 612-2490, E-mail: bbl@berkeley.edu \\ Armen Der Kiureghian \\ Taisei Professor of Civil Engineering Emeritus, \\ University of California, Berkeley, CA 94720. E-mail: adk@ ce.berkeley.edu \\ President, American University of Armenia, Yerevan, Armenia, E-mail: President@aua.am
}

\begin{abstract}
Operational modal analysis is the primary tool for modal parameter identification in civil engineering. Bayesian statistics offers an ideal framework for analyzing uncertainties associated with the identified modal parameters. However, the exact Bayesian formulation is usually intractable due to the high computational demand in obtaining the posterior distributions of modal parameters. In this paper, the variational Bayes method is employed to provide an approximate solution. Unlike the Laplace approximation and Monte Carlo sampling, the variational Bayes approach provides a gradient-free algorithm to analytically approximate the posterior distributions. Working with the state-space representation of a dynamical system, the variational Bayes approach for identification of modal parameters is derived by ignoring statistical correlation between latent variables and the model parameters. In this approach, the joint distribution of the state-transition and observation matrices as well as the joint distribution of the process noise and measurement error are firstly calculated analytically using conjugate priors. The distribution of modal parameters is extracted from these obtained joint distributions using a first-order Taylor series expansion. A robust implementation of the method is discussed by using square-root filtering and Cholesky decomposition. The proposed approach is illustrated by its application to an example mass-spring system and the One Rincon Hill Tower in San Francisco.
\end{abstract}

Keywords: Operational modal analysis, Variational Bayes, System identification, State space model, Linear time-invariant system

\section{Introduction}

Modal parameters, i.e. modal frequencies, damping ratios and mode shapes, are characteristic properties of linear structural models. They are directly related to the mass and stiffness distributions, which are influenced by changes in support and continuity conditions as well as material properties. Identification of modal parameters has become a standard tool for model updating [1], structural control [2], damage detection [3] and condition assessment [4].

A controlled dynamic test usually yields accurate estimates of the modal parameters, but it is seldom applicable to civil structures due to their large size, high load capacity, and noisy operational conditions. Therefore, operational modal analysis (OMA), which utilizes only the stochastic dynamic response, has become the primary modal testing method in civil engineering [5]. The basic assumption in OMA is that the 
sources of excitation are broad-band stochastic processes adequately modeled by band-limited white noise. Since the main sources of excitation in civil structures are wind, traffic, ground tremor and low-magnitude earthquakes, this assumption is generally satisfied.

Many identification methods have been proposed for OMA, but most provide only point estimates. These include NExT-type methods [6], stochastic subspace identification (SSI) [7], the prediction error method [8], FDD-type methods [9] and output-only LSCF-type methods [10]. Since the signal-to-noise ratio cannot be directly controlled and various uncertainties are present in different stages of OMA, uncertainties associated with the identified modal parameters is of primary concern. The impact of uncertainties on operational modal identification has been studied in Ref. [11], where they show that both aleatory and epistemic uncertainties have a significant influence on OMA. In light of this finding, many researchers have improved some of the original algorithms to provide the uncertainty information. Lam \& Mevel [12] derived the confidence interval for the Eigensystem-Realization-Algorithm based on perturbation analysis; Reynders et al. [13] and Dohler et al. [14] developed the confidence intervals for subspace identification; Vu \& Thomas [15] obtained the variance of modal parameters from a vector autoregression model.

The statistical learning method provides a more appropriate approach to extract uncertainty information because all kinds of uncertainties can be explicitly and systematically treated within the same framework [16]. Related approaches include maximum likelihood estimation (MLE) $[17,18]$ and the Bayesian approach $[19,20]$. In MLE, the confidence interval cannot be derived directly and only an asymptotic approximation of the distribution can be derived based on the law of large numbers. In the Bayesian approach, the modal parameters are regarded as random variables, so that their probability distributions can be determined given the measured data and modelling assumptions. From the authors' perspective, the Bayesian approach is perfect for uncertainty quantification in OMA; however, it is often intractable to calculate marginal distributions of the modal parameters. Given the difficulty in directly using the posterior distribution, researchers have mainly followed two methods to approximate the posterior distribution. Both Yuen \& Katafygiotis [19] and $\mathrm{Au}$ [20] applied the Laplace approximation assuming that modal parameters follow a multivariate normal distribution, in spite of the nonlinear relation between modal parameters and measured data. Monte Carlo sampling (MCS) is the other technique used for system identification [21], but it requires massive computing resources if the distributions of parameters are to be well approximated.

Unlike the works of Yuen \& Katafygiotis [19] and Au [20] that focus on the modal parameters directly, we separate the modal identification into two steps: first estimating a stochastic state-space model (SSM) $M(\boldsymbol{\theta})$ and then extracting the modal parameters $\boldsymbol{\eta}$ from $\boldsymbol{\theta}$. The main advantage of this formulation is that inference of parameters $\boldsymbol{\theta}$ in the stochastic SSM from the measured data $\boldsymbol{Y}$ is much easier than inference of the modal parameters $\boldsymbol{\eta}$. Once the posterior distribution of $\boldsymbol{\theta}$ has been inferred, distributions of modal parameters $\boldsymbol{\eta}$ are easily recovered by a first-order Taylor expansion approximation. Therefore, the basic problem lies in inferring the stochastic SSM parameters $\boldsymbol{\theta}$. The proposed variational Bayes approach provides a third alternative for approximately determining the posterior distribution of model parameters.

Variational Bayes (VB) is an approach to analytically approximate the posterior distribution of parameters. Its fundamental principle is to find a surrogate distribution close to the true posterior while making inference tractable. Beal [22] applied the VB framework to identify a SSM, in which, however, the prior setting does not allow invariance under coordinate transformation and cannot be used iteratively. In the area of control, Fujimoto et al. [23] introduced a conjugate prior for the full covariance matrix to fix the aforementioned 
drawbacks for the input-output system identification. In all previous formulations [22, 23], the statetransition matrix and the observation matrix, as well as the process and measurement noise, are assumed to be statistically independent. However, when we use acceleration measurements to identify the modal parameters, the observation matrix consists of mass, stiffness and damping terms as does the state-transition matrix. Therefore, the state-transition and observation matrices in general are statistically dependent. Focusing on OMA, we formulate a new VB algorithm to consider the aforementioned statistical dependencies. By introducing the matrix-variate distribution [24], the derivation becomes much simpler than that in previous studies. Given a matrix normal, inverse Wishart (MNIW) prior distribution for the state transition, observation and covariance matrices and assuming multivariate normal distribution for the initial state, measurement and model errors, the posterior marginal distribution of the state transition and observation matrix can be theoretically derived to be the matrix variate- $t$ distribution. Furthermore, a numerically robust implementation is presented to make this algorithm more reliable and capable for highdimension modal identification.

Using the VB algorithm we can obtain the posterior distribution of the state-transition and observation matrices; however, there is no closed-form solution for the distribution of the modal parameters. In this paper, a first-order Taylor series expansion at the mean values of the state-transition and observation matrices is applied, resulting in a multivariate lognormal distribution for the frequencies and damping ratios and a multivariate normal distribution for the mode shapes. This is because the matrix variate- $t$ distribution converges to a matrix normal distribution when the degree of freedom tends to infinity, which happens when the sequence of data is long. A new way to calculate the Jacobian matrix is introduced, especially for the mode shapes.

\section{Variational Bayes}

In this section, we present a broad overview of the variational Bayes algorithm in a general setting. The fundamental idea of VB is to approximate the posterior distribution of the unknown parameters by a tractable surrogate distribution that is suitable to infer the parameters in the latent variable model. To be more precise, consider a simple Bayesian linear normal model,

$$
\boldsymbol{Y}=\boldsymbol{\Theta} \boldsymbol{X}+\boldsymbol{\epsilon}
$$

in which $\boldsymbol{Y} \in \mathbb{R}^{m}$ is the set of observed variables, $\boldsymbol{X} \in \mathbb{R}^{n}$ is the set of latent variables, $\boldsymbol{\epsilon} \in \mathbb{R}^{m}$ represents measurement errors, which are assumed to be normal, and $\boldsymbol{\Theta} \in \mathbb{R}^{m \times n}$ is the matrix of uncertain model parameters. The above model can also be represented as a probabilistic graphical model (PGM), as shown in Fig. 1. Each node is associated with a set of random variables and directed links refer to conditional dependencies.

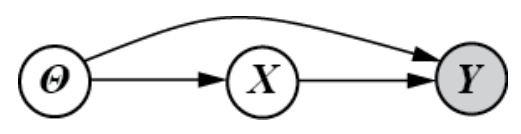

Fig. 1 Probabilistic graphical model of Bayesian linear normal model.

The problem is to infer the posterior distribution $p_{\boldsymbol{\theta} \mid \boldsymbol{Y}}(\boldsymbol{\theta} \mid \boldsymbol{y})$, given the data $\boldsymbol{y}$ and the prior distribution $\pi_{\boldsymbol{\theta} \boldsymbol{X}}(\boldsymbol{\theta}, \boldsymbol{x})$. Since $\boldsymbol{\Theta}$ and $\boldsymbol{X}$ are coupled in this model, there are rare cases where one can find an analytical 
solution to the problem. Instead, we try to find a surrogate distribution $q_{\boldsymbol{\theta} X}^{*}(\boldsymbol{\theta}, \boldsymbol{x})$ from a predetermined family $\mathbb{Q}$ to minimize the distance from the true posterior distribution $p_{\boldsymbol{\theta} X \mid Y}(\boldsymbol{\theta}, \boldsymbol{x} \mid \boldsymbol{y})$ in the sense of the Kullback-Leibler (KL) divergence measure [16]. Formally,

$$
q_{\boldsymbol{\theta} \boldsymbol{X}}^{*}(\boldsymbol{\theta}, \boldsymbol{x})=\arg \min _{q_{\boldsymbol{\theta} X}} \mathrm{KL}\left[q_{\boldsymbol{\theta} \boldsymbol{X}}(\boldsymbol{\theta}, \boldsymbol{x}) \| p_{\boldsymbol{\theta} \boldsymbol{X} \mid \boldsymbol{Y}}(\boldsymbol{\theta}, \boldsymbol{x} \mid \boldsymbol{y})\right]=\arg \min _{q_{\boldsymbol{\theta} X}} \iint q_{\boldsymbol{\theta} \boldsymbol{X}}(\boldsymbol{\theta}, \boldsymbol{x}) \ln \frac{q_{\boldsymbol{\theta} X}(\boldsymbol{\theta}, \boldsymbol{x})}{p_{\boldsymbol{\theta} \mid \boldsymbol{Y}}(\boldsymbol{\theta}, \boldsymbol{x} \mid \boldsymbol{y})} d \boldsymbol{\theta} d \boldsymbol{x}
$$

It is usually difficult to evaluate the KL divergence in Eqn. (2), so we use an alternative method to minimize it without its direct evaluation. The log marginal likelihood of the observed variables $\boldsymbol{y}$ is given by

$$
\begin{aligned}
& \ln p_{\boldsymbol{Y}}(\boldsymbol{y})=\iint q_{\boldsymbol{\theta X}}(\boldsymbol{\theta}, \boldsymbol{x}) \ln p_{\boldsymbol{Y}}(\boldsymbol{y}) d \boldsymbol{\theta} d \boldsymbol{x}=\iint q_{\boldsymbol{\theta} \boldsymbol{X}}(\boldsymbol{\theta}, \boldsymbol{x}) \ln \frac{p_{\boldsymbol{\theta X Y}(\boldsymbol{\theta}, \boldsymbol{x}, \boldsymbol{y})}}{\left.p_{\boldsymbol{\theta} X \mid \boldsymbol{Y}} \boldsymbol{\theta}, \boldsymbol{x} \mid \boldsymbol{y}\right)} d \boldsymbol{\theta} d \boldsymbol{x} \\
& =\iint q_{\boldsymbol{\theta} X}(\boldsymbol{\theta}, \boldsymbol{x}) \ln \frac{q_{\boldsymbol{\theta} X}(\boldsymbol{\theta}, \boldsymbol{x})}{p_{\boldsymbol{\theta} X \mid Y}(\boldsymbol{\theta}, \boldsymbol{x} \mid \boldsymbol{y})} d \boldsymbol{\theta} d \boldsymbol{x}+\iint q_{\boldsymbol{\theta} X}(\boldsymbol{\theta}, \boldsymbol{x}) \ln \frac{p_{Y \mid \boldsymbol{\theta} X}(\boldsymbol{y} \mid \boldsymbol{\theta}, \boldsymbol{x}) \pi_{\boldsymbol{\theta} X}(\boldsymbol{\theta}, \boldsymbol{x})}{q_{\boldsymbol{\theta} X}(\boldsymbol{\theta}, \boldsymbol{x})} d \boldsymbol{\theta} d \boldsymbol{x} \\
& \triangleq \mathrm{KL}\left[q_{\boldsymbol{\theta} \boldsymbol{X}}(\boldsymbol{\theta}, \boldsymbol{x}) \| p_{\boldsymbol{\theta X} \mid \boldsymbol{Y}}(\boldsymbol{\theta}, \boldsymbol{x} \mid \boldsymbol{y})\right]+\mathrm{F}\left[q_{\boldsymbol{\theta} \boldsymbol{X}}(\boldsymbol{\theta}, \boldsymbol{x})\right]
\end{aligned}
$$

where $\mathrm{F}\left[q_{\boldsymbol{\theta} \boldsymbol{X}}(\boldsymbol{\theta}, \boldsymbol{x})\right]$, known as "free energy"[16], is the last integral in the penultimate line in Eq. (3). Since the log marginal likelihood must remain constant for a given model, minimizing the KL divergence is equivalent to maximizing the free energy $\mathrm{F}\left[q_{\boldsymbol{\theta X}}(\boldsymbol{\theta}, \boldsymbol{x})\right]$. On the other hand, since the KL divergence is nonnegative, the free energy works as a lower bound of the log marginal likelihood; therefore, we can regard the VB as maximizing the marginal likelihood by increasing its lower bound.

As is often the case in Bayesian inference, the parameters and latent variables are coupled, leading to a problem that is analytically unsolvable. To overcome this difficulty, the mean-field approximation [16] is introduced to make an independence assumption between $\boldsymbol{\Theta}$ and $\boldsymbol{X}$, i.e. $q_{\boldsymbol{\theta} \boldsymbol{X}}(\boldsymbol{\theta}, \boldsymbol{x})=q_{\boldsymbol{\theta}}(\boldsymbol{\theta}) q_{\boldsymbol{X}}(\boldsymbol{x})$ is assumed. This gives a good approximation when the dependence between the latent variables and parameters is mild. Although this assumption may seem drastic, one may think of it as replacing the stochastic dependence between $\boldsymbol{\Theta}$ and $\boldsymbol{X}$ by deterministic dependencies between their relevant moments [22]. Following this assumption and assigning independent prior distributions for $\boldsymbol{\Theta}$ and $\boldsymbol{X}$, we have

$$
\begin{aligned}
\mathrm{F}\left[q_{\boldsymbol{\theta} \boldsymbol{X}}(\boldsymbol{\theta}, \boldsymbol{x})\right] & \approx \mathrm{F}\left[q_{\boldsymbol{\theta}}(\boldsymbol{\theta}), q_{\boldsymbol{X}}(\boldsymbol{x})\right] \\
& =\iint q_{\boldsymbol{\Theta}}(\boldsymbol{\theta}) q_{\boldsymbol{X}}(\boldsymbol{x}) \ln p_{\boldsymbol{Y} \mid \boldsymbol{\theta} \boldsymbol{X}}(\boldsymbol{y} \mid \boldsymbol{\theta}, \boldsymbol{x}) d \boldsymbol{\theta} d \boldsymbol{x}-\int q_{\boldsymbol{\theta}}(\boldsymbol{\theta}) \ln \frac{q_{\boldsymbol{\theta}}(\boldsymbol{\theta})}{\pi_{\boldsymbol{\theta}}(\boldsymbol{\theta})} d \boldsymbol{\theta}-\int q_{\boldsymbol{X}}(\boldsymbol{x}) \ln \frac{q_{X}(\boldsymbol{x})}{\pi_{X}(\boldsymbol{x})} d \boldsymbol{x} \\
& =\mathrm{E}_{\boldsymbol{\Theta} \boldsymbol{X}}\left[\ln p_{\boldsymbol{Y} \mid \boldsymbol{\theta} \boldsymbol{X}}(\boldsymbol{y} \mid \boldsymbol{\theta}, \boldsymbol{x})\right]-\mathrm{KL}\left[q_{\boldsymbol{\theta}}(\boldsymbol{\theta}) \| \pi_{\boldsymbol{\theta}}(\boldsymbol{\theta})\right]-\mathrm{KL}\left[q_{\boldsymbol{X}}(\boldsymbol{x}) \| \pi_{\boldsymbol{X}}(\boldsymbol{x})\right]
\end{aligned}
$$

where $\mathrm{E}_{\boldsymbol{\Theta X}}[\cdot]$ denotes the expectation operator with respect to the joint distribution of $\boldsymbol{\Theta}$ and $\boldsymbol{X}$. Thus, in order to maximize the free energy $\mathrm{F}\left[q_{\boldsymbol{\theta}}(\boldsymbol{\theta}), q_{\boldsymbol{X}}(\boldsymbol{x})\right]$, we have to reduce the KL divergences between the approximating and prior distributions and simultaneously increase the expected log conditional likelihood. The free energy is a functional of the posterior distributions $q_{\boldsymbol{\theta}}(\boldsymbol{\theta})$ and $q_{\boldsymbol{X}}(\boldsymbol{x})$ and the prior distributions $\pi_{\boldsymbol{\theta}}(\boldsymbol{\theta})$ and $\pi_{\boldsymbol{X}}(\boldsymbol{x})$. Therefore, we can iteratively maximize it with respect the four distributions, which is essentially a coordinate ascent method in the function space. The following theorem describes the steps in the updating formula for VB inference, which are illustrated in Fig.2.

Theorem 1. Variational Bayesian Expectation Maximization Prior-updating (VB-EMP) 
Let $\mathcal{M}(\boldsymbol{\Theta})$ be a statistical model with parameters $\boldsymbol{\Theta}$ giving rise to an observed data set $\boldsymbol{y}$ with corresponding latent variables $\boldsymbol{X}$. The free energy $\mathrm{F}\left[q_{\boldsymbol{\Theta}}(\boldsymbol{\theta}), q_{\boldsymbol{X}}(\boldsymbol{x})\right]$ given in Eqn. (4) can be iteratively maximized by performing the following updates, using superscript $t$ to denote iteration step:

VB Expectation (VBE): $\quad q_{\boldsymbol{X}}^{(t+1)}(\boldsymbol{x})=Z_{\boldsymbol{X}}^{-1} \pi_{\boldsymbol{X}}^{(t)}(\boldsymbol{x}) \exp \left[\int q_{\boldsymbol{\Theta}}^{(t)}(\boldsymbol{\theta}) \ln p_{\boldsymbol{Y} \mid \boldsymbol{\theta} \boldsymbol{X}}(\boldsymbol{y} \mid \boldsymbol{\theta}, \boldsymbol{x}) d \boldsymbol{\theta}\right]$

VB Maximization $(\mathrm{VBM}): \quad q_{\boldsymbol{\Theta}}^{(t+1)}(\boldsymbol{\theta})=Z_{\boldsymbol{\Theta}}^{-1} \pi_{\boldsymbol{\Theta}}^{(t)}(\boldsymbol{\theta}) \exp \left[\int q_{\boldsymbol{X}}^{(t)}(\boldsymbol{x}) \ln p_{\boldsymbol{Y} \mid \boldsymbol{\theta} \boldsymbol{X}}(\boldsymbol{y} \mid \boldsymbol{\theta}, \boldsymbol{x}) d \boldsymbol{x}\right]$

VB Prior-updating (VBP): $\quad \pi_{\boldsymbol{\theta}}^{(t+1)}(\boldsymbol{\theta})=q_{\boldsymbol{\Theta}}^{(t+1)}(\boldsymbol{\theta}) ; \quad \pi_{\boldsymbol{X}}^{(t+1)}(\boldsymbol{x})=q_{\boldsymbol{X}}^{(t+1)}(\boldsymbol{x})$.

where $Z_{\boldsymbol{X}}$ and $Z_{\boldsymbol{\Theta}}$ are normalizing constants. It is noted that each step increases $\mathrm{F}\left[q_{\boldsymbol{\Theta}}(\boldsymbol{\theta}), q_{\boldsymbol{X}}(\boldsymbol{x})\right]$ monotonically and the sequence converges to a local maximum.

A general proof of the above theorem is presented by Beal [22].

In addition to the monotonic convergence, for the exponential family of distributions, Wang \& Titterington [25] have shown that the VB-EMP algorithm is statistically consistent, i.e. it converges to the true value in an asymptotic sense, provided the starting distributions are sufficiently close to the true solutions. The drawbacks of the method are: (a) it is impossible to represent multimodal posteriors because we approximate the posterior by only one approximating distribution; (b) the method is sensitive to the starting point, so that one must provide a good initial guess; (c) since the prior is updated in each iteration as in the empirical Bayes method [26], the variability in the posterior distribution tends to be underestimated [27].

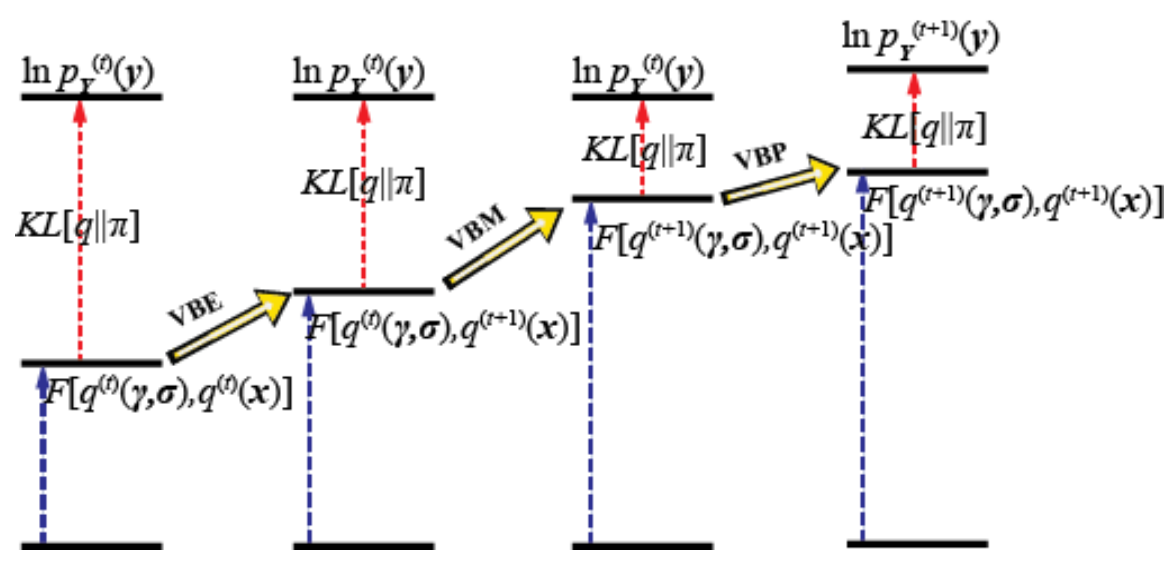

Fig. 2 Variational Bayes EMP.

\section{Stochastic SSM}

For a discretized linear time-invariant dynamical system with $N_{d}$ degrees of freedom (DOFs), the equation of motion is represented as

$$
\boldsymbol{M} \ddot{\boldsymbol{u}}(t)+\boldsymbol{C}_{d} \dot{\boldsymbol{u}}(t)+\boldsymbol{K u}(t)=\boldsymbol{P} \boldsymbol{f}(t)-\boldsymbol{M} \ddot{\boldsymbol{u}}_{g}(t) ; \quad \boldsymbol{u}(0)=\boldsymbol{u}_{0}, \dot{\boldsymbol{u}}(0)=\dot{\boldsymbol{u}}_{0}
$$

where $\boldsymbol{M} \in \mathbb{R}^{N_{d} \times N_{d}}, \boldsymbol{C}_{d} \in \mathbb{R}^{N_{d} \times N_{d}}$ and $\boldsymbol{K} \in \mathbb{R}^{N_{d} \times N_{d}}$ are the mass, damping and stiffness matrices, respectively; $\boldsymbol{u}(t) \in \mathbb{R}^{N_{d}}, \dot{\boldsymbol{u}}(t) \in \mathbb{R}^{N_{d}}$ and $\ddot{\boldsymbol{u}}(t) \in \mathbb{R}^{N_{d}}$ are the nodal displacement, velocity and acceleration 
responses relative to the ground, respectively; $\boldsymbol{f}(t) \in \mathbb{R}^{N_{f}}$ is the external force vector; $\boldsymbol{P} \in \mathbb{R}^{N_{d} \times N_{f}}$ is the load coefficient matrix representing the spatial influence of the external force; $\ddot{\boldsymbol{u}}_{g}(t) \in \mathbb{R}^{N_{g}}$ is the ground acceleration vector, and $\boldsymbol{l} \in \mathbb{R}^{N_{d} \times N_{g}}$ is the corresponding influence matrix. $\boldsymbol{u}_{0} \in \mathbb{R}^{N_{d}}$ and $\dot{\boldsymbol{u}}_{0} \in \mathbb{R}^{N_{d}}$ are the initial relative displacement and velocity vectors, respectively. Note that we are considering both an external force and a base motion. This is because under operating conditions the structure is usually subject to the effects of wind, traffic, ground tremor and small magnitude earthquakes. For system identification, it is convenient to convert Eqn. (5) into a continuous-time state-space model:

$$
\dot{\boldsymbol{X}}(t)=\boldsymbol{A}_{c} \boldsymbol{X}(t)+\boldsymbol{B}_{c f} \boldsymbol{f}(t)-\boldsymbol{B}_{c g} \ddot{\boldsymbol{u}}_{g}(t)
$$

with $\boldsymbol{X}(t)=\left[\begin{array}{l}\boldsymbol{u}(t) \\ \dot{\boldsymbol{u}}(t)\end{array}\right], \boldsymbol{A}_{c}=\left[\begin{array}{cc}\mathbf{0}_{N_{d}} & \boldsymbol{I}_{N_{d}} \\ -\boldsymbol{M}^{-1} \boldsymbol{K} & -\boldsymbol{M}^{-1} \boldsymbol{C}_{d}\end{array}\right], \boldsymbol{B}_{c f}=\left[\begin{array}{c}\mathbf{0}_{N_{d} \times N_{f}} \\ \boldsymbol{M}^{-1} \boldsymbol{P}\end{array}\right]$, and $\boldsymbol{B}_{c g}=\left[\begin{array}{c}\mathbf{0}_{N_{d} \times N_{g}} \\ \boldsymbol{l}\end{array}\right]$.

The state vector $\boldsymbol{X}(t) \in \mathbb{R}^{N_{s}}$ is assumed as a latent variable, where $N_{s}=2 N_{d}$ is the model order. The output of interest is expressed as

$$
\boldsymbol{Y}(t)=\boldsymbol{C} \boldsymbol{X}(t)+\boldsymbol{D}_{f} \boldsymbol{f}(t)-\boldsymbol{D}_{g} \ddot{\boldsymbol{u}}_{g}(t)
$$

where the observed variables $\boldsymbol{Y}(t) \in \mathbb{R}^{N_{O}}$ are any quantities that directly relate to the nodal displacements or velocities. In practice, nodal accelerations are easy to measure with high resolution. In that case, $\boldsymbol{C}=$ $\boldsymbol{S}_{o}\left[-\boldsymbol{M}^{-1} \boldsymbol{K}-\boldsymbol{M}^{-1} \boldsymbol{C}_{d}\right], \boldsymbol{D}_{f}=\boldsymbol{S}_{o} \boldsymbol{M}^{-1} \boldsymbol{P}$ and $\boldsymbol{D}_{g}=\boldsymbol{S}_{o} \boldsymbol{l}$, where $\boldsymbol{S}_{o} \in \mathbb{R}^{N_{o} \times N_{S}}$ is a selection matrix that defines the $N_{o}\left(N_{o} \leq N_{S}\right)$ DOFs of the structure at which measurements are made.

Employing a zero-order-hold ( $\mathrm{ZOH})$ assumption [28] on the input, we discretize Eqns. (6) and (7) into the form

$$
\begin{gathered}
\boldsymbol{X}_{k+1}=\boldsymbol{A} \boldsymbol{X}_{k}+\boldsymbol{B}_{f} \boldsymbol{f}_{k}-\boldsymbol{B}_{g} \ddot{\boldsymbol{u}}_{g, k} \\
\boldsymbol{Y}_{k}=\boldsymbol{C} \boldsymbol{X}_{k}+\boldsymbol{D}_{f} \boldsymbol{f}_{k}-\boldsymbol{D}_{g} \ddot{\boldsymbol{u}}_{g, k}
\end{gathered}
$$

in which $\boldsymbol{X}_{k} \in \mathbb{R}^{N_{s}}$ and $\boldsymbol{Y}_{k} \in \mathbb{R}^{N_{o}}$ are the recorded responses at time step $k T_{s}$ for $k=1,2, \ldots, N, \boldsymbol{A}=e^{\boldsymbol{A}_{c} T_{s}}$, $\boldsymbol{B}_{f}=\left(\boldsymbol{A}-I_{N_{S}}\right) \boldsymbol{A}_{c}^{-1} \boldsymbol{B}_{c f}$, and $\boldsymbol{B}_{g}=\left(\boldsymbol{A}-I_{N_{S}}\right) \boldsymbol{A}_{c}^{-1} \boldsymbol{B}_{c g} . T_{s}$ is the sampling period; it should be much smaller than half of the fundamental period of the structure in order for the $\mathrm{ZOH}$ assumption to hold.

In OMA, the modal parameters are extracted from the dynamic response to unmeasured operational forces. Therefore, the stochastic force $\boldsymbol{f}_{k}$ and base motion $\ddot{\boldsymbol{u}}_{g, k}$ remain unknown. In this work they are assumed to be band-limited Gaussian white-noise processes, i.e. their power spectrum density functions are flat over the frequency range relevant to the structural response. Besides these unknown forces, the system is always subject to model uncertainty and measurement error. Combining all these effects, we model the total uncertainties by two noise terms $\boldsymbol{w}_{k}$ and $\boldsymbol{v}_{k}$, resulting in the stochastic state-space model

$$
\left[\begin{array}{c}
\boldsymbol{X}_{k+1} \\
\boldsymbol{Y}_{k}
\end{array}\right]=\left[\begin{array}{c}
\boldsymbol{A} \\
\boldsymbol{C}
\end{array}\right] \boldsymbol{X}_{k}+\left[\begin{array}{c}
\boldsymbol{W}_{k} \\
\boldsymbol{v}_{k}
\end{array}\right]
$$

where $\left[\begin{array}{ll}\boldsymbol{w}_{k}^{\mathrm{T}} & \boldsymbol{v}_{k}^{\mathrm{T}}\end{array}\right]^{\mathrm{T}}$ is modeled as a vector of zero-mean Gaussian band-limited white-noise processes with unknown covariance matrix $\boldsymbol{\Sigma}=\left[\begin{array}{cc}\boldsymbol{Q} & \boldsymbol{S} \\ \boldsymbol{S}^{\mathrm{T}} & \boldsymbol{R}\end{array}\right]$ for $k=1,2, \ldots, N$. More specifically, $\boldsymbol{w}_{k}$ represents the joint 
effects of unknown external forces $\boldsymbol{f}_{k}$, ground accelerations $\ddot{\boldsymbol{u}}_{g, k}$, and the model error, while $\boldsymbol{v}_{k}$ stands for the joint effects of the external forces, ground accelerations, and measurement error. Since both $\boldsymbol{w}_{k}$ and $\boldsymbol{v}_{k}$ follow the multivariate normal distribution, if we assume the initial state $\boldsymbol{X}_{1}$ is also multivariate normal, the overall dynamical process becomes Gaussian and can be depicted by the PGM in Figure 3.

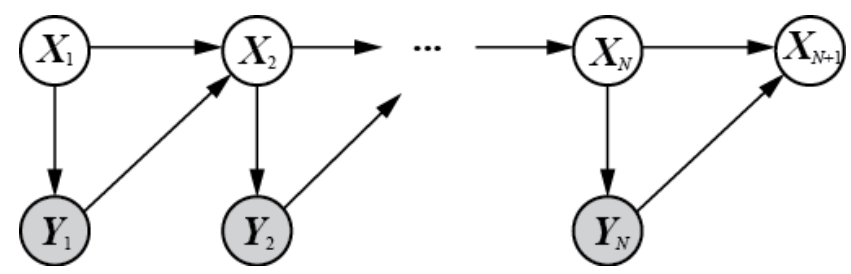

Fig. 3 Probabilistic graphical model of stochastic SSM with deterministic parameters

The model in Figure 3 has the Markov property

$$
p_{\boldsymbol{Z}_{k} \mid \boldsymbol{X}_{1: k}}\left(\boldsymbol{z}_{k} \mid \boldsymbol{x}_{1: k}\right)=p_{\boldsymbol{Z}_{k} \mid \boldsymbol{X}_{k}}\left(\mathbf{z}_{k} \mid \boldsymbol{x}_{k}\right)=\mathrm{N}\left(\boldsymbol{\Gamma} \boldsymbol{x}_{k}, \boldsymbol{\Sigma}\right)
$$

where we have used the notation $\boldsymbol{X}_{1: k}=\left\{\boldsymbol{X}_{1}, \boldsymbol{X}_{2}, \ldots, \boldsymbol{X}_{k}\right\}, \boldsymbol{Z}_{k}=\left[\begin{array}{ll}\boldsymbol{X}_{k+1}^{\mathrm{T}} & \boldsymbol{Y}_{k}^{\mathrm{T}}\end{array}\right]^{\mathrm{T}}, \boldsymbol{\Gamma}=\left[\begin{array}{ll}\boldsymbol{A}^{\mathrm{T}} & \boldsymbol{C}^{\mathrm{T}}\end{array}\right]^{\mathrm{T}}$ and $\mathrm{N}\left(\boldsymbol{\Gamma} \boldsymbol{x}_{k}, \boldsymbol{\Sigma}\right)$ denotes the probability density function (PDF) of a multivariate normal distribution with mean $\boldsymbol{\Gamma} \boldsymbol{x}_{k}$ and covariance matrix $\boldsymbol{\Sigma}$.

It is well-known that the state transition matrix $\boldsymbol{A}$ and observation matrix $\boldsymbol{C}$ are unique up to a similarity transformation [29]. That is, for only one measured input-output pair or only one measured output, there could possibly be infinite pairs of $\boldsymbol{A}$ and $\boldsymbol{C}$ satisfying Eqn. (10). However, the modal parameters of the structure are invariant under a similarity transformation; therefore, they can be uniquely determined from the measured data.

The modal parameters are extracted by applying the following steps [5]:

(1) Apply the eigenvalue decomposition $\boldsymbol{A}=\boldsymbol{\Psi} \boldsymbol{\Lambda} \boldsymbol{\Psi}^{-1}$;

(2) Since the eigen-solutions occur in complex conjugate pairs, partition the eigenvalue and eigenvector matrices according to

$$
\boldsymbol{\Lambda}=\left[\begin{array}{ll}
\lambda & \mathbf{0} \\
\mathbf{0} & \bar{\lambda}
\end{array}\right], \boldsymbol{\Psi}=\left[\begin{array}{ll}
\boldsymbol{\psi} & \overline{\boldsymbol{\psi}}
\end{array}\right]
$$

(3) Extract the $i$-th modal frequency and modal damping ratio as

$$
\lambda_{c i}=\ln \lambda_{i} / T_{s}, f_{i}=\left|\lambda_{c i}\right| / 2 \pi, \xi_{i}=-\operatorname{Re}\left(\lambda_{c i}\right) /\left|\lambda_{c i}\right|
$$

where $\lambda_{i}$ is the $i$-th diagonal element of $\lambda$.

(4) Calculate the $i$-th mode shape:

$$
\phi_{i}=C \psi_{i}
$$

where $\boldsymbol{\psi}_{i}$ is the $i$-th column of eigenvector matrix $\boldsymbol{\psi}$.

The obtained mode shape $\boldsymbol{\phi}_{i}$ in Eqn. (14) generally is a complex vector, but the real mode shape is more meaningful to the engineers. Here, a novel way to extract the $i$-th real mode shape is proposed:

1) Define a matrix $\boldsymbol{\varphi}_{i}=\left[\operatorname{Re}\left(\boldsymbol{\psi}_{i}\right) \operatorname{Im}\left(\boldsymbol{\psi}_{i}\right)\right]$;

2) Take the generalized eigenvalue decomposition:

$$
\boldsymbol{P}_{1} \boldsymbol{\varphi}_{i}^{\mathrm{T}} \boldsymbol{C}^{\mathrm{T}} \boldsymbol{C} \boldsymbol{\varphi}_{i} \boldsymbol{P}_{1} \hat{\mathbf{z}}=\lambda_{\min } \boldsymbol{P}_{2} \boldsymbol{\varphi}_{i}^{\mathrm{T}} \boldsymbol{C}^{\mathrm{T}} \boldsymbol{C} \boldsymbol{\varphi}_{i} \boldsymbol{P}_{2} \hat{\mathbf{z}}
$$


where $\boldsymbol{P}_{1}=\left[\begin{array}{lll}0 & 1 ; 1 & 0\end{array}\right]$ and $\boldsymbol{P}_{2}=\left[\begin{array}{lll}1 & 0 ; 0 & -1\end{array}\right] ; \lambda_{\min }$ is the minimum eigenvalue and $\hat{\boldsymbol{z}}$ is the corresponding eigenvector;

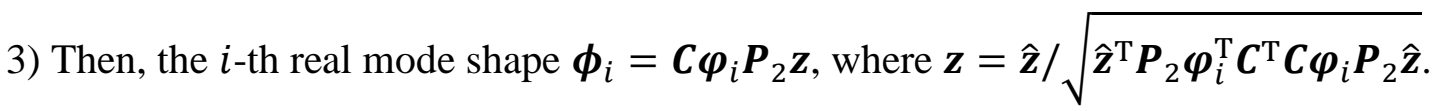

Using the Lagrange multiplier method, we can show that $\mathbf{z}$ is the optimal solution to the following problem

$$
\min _{z_{i}}\left\|\operatorname{Im}\left(z_{i} \boldsymbol{C} \boldsymbol{\psi}_{i}\right)\right\|_{2} \quad \text { s.t. }\left\|\operatorname{Re}\left(z_{i} \boldsymbol{C} \boldsymbol{\psi}_{i}\right)\right\|_{2}=1
$$

in which $z_{i}=z_{1}+z_{2} \sqrt{-1}, z_{1}$ and $z_{2}$ are the first and second element of $\mathbf{z}$. 'Im' and 'Re' denote the imaginary and real parts of a complex vector, respectively; ' $\|\cdot\|_{2}$ ' stands for the $L_{2}$ vector norm. In addition, it is easy to show that $\min \left\|\operatorname{Im}\left(z_{i} \boldsymbol{C} \boldsymbol{\psi}_{i}\right)\right\|_{2}=\lambda_{\text {min }}$, which can be used as an index to distinguish spurious modes, if we presume that the physical mode shapes are real vectors.

\section{Application of VB for Stochastic SSM}

In the preceding section, we transformed the equation of motion of the LTI system into a stochastic SSM form. In this section, we consider the problem of estimating the parameters $\boldsymbol{\Theta}=\{\boldsymbol{\Gamma}, \boldsymbol{\Sigma}\}$ of the stochastic SSM, given the observed sequence $\boldsymbol{y}_{1: N}=\left\{\boldsymbol{y}_{1}, \ldots, \boldsymbol{y}_{N}\right\}$. Note that matrices $\boldsymbol{A}$ and $\boldsymbol{C}$ in Eqn. (10) share common terms, leading them to be statistically dependent; therefore, the $\boldsymbol{\Gamma}$ is considered as a matrix random variable. Since both $\boldsymbol{w}_{k}$ and $\boldsymbol{v}_{k}$ include the effects of unmeasured external forces and ground accelerations, they should be statistically correlated, yielding a full covariance matrix $\boldsymbol{\Sigma}$. These aspects differentiate this study from all previous studies $[22,23]$.

With parameters $\boldsymbol{\Theta}$ considered as random variables, the new PGM is described as Fig. 4. It is evident that parameters $\boldsymbol{\Theta}$ and latent variables $\boldsymbol{X}_{1: N+1}$ are coupled, leading to the loss of Markov property. Under these conditions, exact Bayesian inference becomes analytically intractable. Hence, we apply the variational Bayes framework to solve this problem, i.e. we find the factorized distribution $q_{\boldsymbol{\Theta}}(\boldsymbol{\theta}) q_{\boldsymbol{X}_{1: N+1}}\left(\boldsymbol{x}_{1: N+1}\right)$ to approximate the true posterior distribution $p_{\boldsymbol{\Theta} \boldsymbol{X}_{1: N+1} \mid \boldsymbol{Y}_{1: N}}\left(\boldsymbol{\theta}, \boldsymbol{x}_{1: N+1} \mid \boldsymbol{y}_{1: N}\right)$.

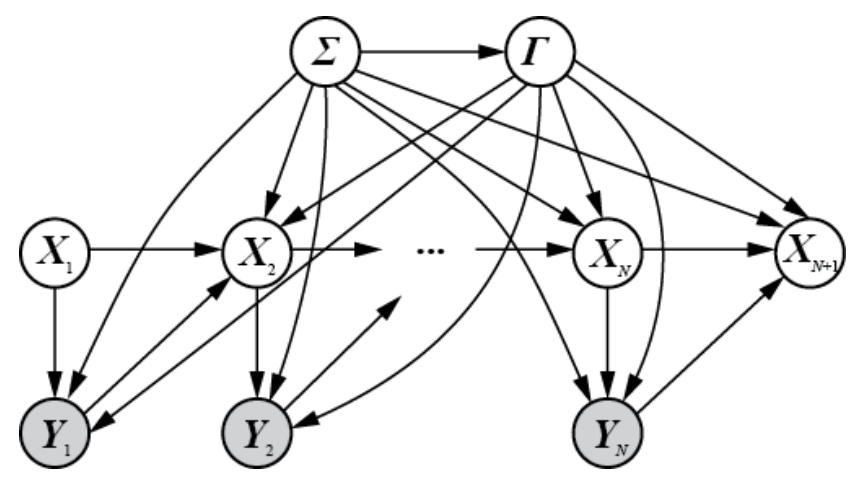

Fig. 4 Probabilistic graphical model of stochastic SSM with randomized parameters

Before applying Theorem 1, we define conjugate priors for parameters $\boldsymbol{\Theta}$. Conjugate priors are commonly used in Bayesian statistics in order to obtain closed-form solutions for posteriors. For $\boldsymbol{\Theta}$ in the PGM in Fig. 4, we assume a MNIW prior distribution [24], i.e.

$$
\pi_{\Theta}(\boldsymbol{\theta})=\pi_{\boldsymbol{\Gamma} \mid \Sigma}(\boldsymbol{\gamma} \mid \boldsymbol{\sigma}) \pi_{\boldsymbol{\Sigma}}(\boldsymbol{\sigma})=\mathrm{MN}\left(\boldsymbol{\mu}_{\boldsymbol{\Gamma}}, \boldsymbol{\Sigma}, \boldsymbol{\Pi}\right) \operatorname{IW}(d, \boldsymbol{D})
$$


In the above, $\operatorname{MN}\left(\boldsymbol{\mu}_{\boldsymbol{\Gamma}}, \boldsymbol{\Sigma}, \boldsymbol{\Pi}\right)$ defines the PDF of the matrix normal distribution with mean matrix $\boldsymbol{\mu}_{\boldsymbol{\Gamma}} \in$ $\mathbb{R}^{\left(N_{S}+N_{o}\right) \times N_{S}}$ and left- and right-covariance matrices $\boldsymbol{\Sigma} \in \mathbb{R}^{\left(N_{S}+N_{o}\right) \times\left(N_{S}+N_{o}\right)}$ and $\boldsymbol{\Pi} \in \mathbb{R}^{N_{S} \times N_{S}}$, respectively. $\operatorname{IW}(d, \boldsymbol{D})$ denotes the PDF of the inverse Wishart distribution with $d$ DOFs and scale matrix $\boldsymbol{D} \in$ $\mathbb{R}^{\left(N_{S}+N_{O}\right) \times\left(N_{S}+N_{o}\right)}$. For the reader's convenience, definitions of these distributions are provided in Appendix A. As for the latent variables $\boldsymbol{X}_{1: N+1}$, we only need to specify a prior for $\boldsymbol{X}_{1}$; this information will then automatically propagate through the chain structure of the PGM. Therefore, we specify a multivariate normal distribution for $\boldsymbol{X}_{1}$ with unknown mean $\boldsymbol{\mu}_{1}$ and covariance matrix $\boldsymbol{P}_{1}$, i.e. $\pi_{\boldsymbol{X}_{1}}\left(\boldsymbol{x}_{1}\right)=N\left(\boldsymbol{\mu}_{1}, \boldsymbol{P}_{1}\right)$. Applying the basic procedure of variational Bayes to the more complicated stochastic SSM, we state the following theorem:

Theorem 2. Considering the PGM in Fig. 4, the prior distribution of parameter $\boldsymbol{\Theta}$ in Eqn. (14) and the assumed normal prior of $\boldsymbol{X}_{1}$, for the latent variable $\boldsymbol{X}_{1: N+1}$, observed variable $\boldsymbol{Y}_{1: N}$ and parameters $\boldsymbol{\Theta}$ :

(1) The free energy function $\mathrm{F}\left[q_{\boldsymbol{\Theta}}(\boldsymbol{\theta}), q_{\boldsymbol{X}_{1: N+1}}\left(\boldsymbol{x}_{1: N+1}\right)\right]$ is given by

$$
\begin{aligned}
\mathrm{F}\left[q_{\boldsymbol{\Theta}}(\boldsymbol{\theta}), q_{\boldsymbol{X}_{1: N+1}}\left(\boldsymbol{x}_{1: N+1}\right)\right]= & -\iint q_{\Gamma \boldsymbol{\Sigma}}(\boldsymbol{\gamma}, \boldsymbol{\sigma}) \ln \frac{q_{\Gamma \Sigma}(\gamma, \boldsymbol{\sigma})}{\pi_{\Gamma \Sigma}(\gamma, \boldsymbol{\sigma})} d \boldsymbol{\gamma} d \boldsymbol{\sigma}-\int q_{\boldsymbol{X}_{1: N+1}}\left(\boldsymbol{x}_{1: N+1}\right) \ln \frac{q_{X_{1: N+1}}\left(\boldsymbol{x}_{1: N+1}\right)}{\pi_{X_{1}}\left(\boldsymbol{x}_{1}\right)} d \boldsymbol{x}_{1: N+1} \\
& +\iiint q_{\boldsymbol{I} \Sigma}(\boldsymbol{\gamma}, \boldsymbol{\sigma}) q_{\boldsymbol{X}_{1: N+1}}\left(\boldsymbol{x}_{1: N+1}\right) \sum_{k=1}^{N} \ln p_{\boldsymbol{Z}_{k} \mid \boldsymbol{\Gamma} \boldsymbol{\Sigma} \boldsymbol{X}_{k}}\left(\mathbf{z}_{k} \mid \boldsymbol{\gamma}, \boldsymbol{\sigma}, \boldsymbol{x}_{k}\right) d \boldsymbol{\gamma} d \boldsymbol{\sigma} d \boldsymbol{x}_{k}
\end{aligned}
$$

(2) $\mathrm{F}\left[q_{\boldsymbol{\Theta}}(\boldsymbol{\theta}), q_{\boldsymbol{X}_{1: N+1}}\left(\boldsymbol{x}_{1: N+1}\right)\right]$ is iteratively optimized by performing the following updates, using superscript $t$ to denote iteration step:

VBE step:

$$
\begin{aligned}
& q_{X_{1: N+1}}^{(t+1)}\left(\boldsymbol{x}_{1: N+1}\right)= \\
& Z_{\boldsymbol{X}_{1: N+1}^{-1}}^{-} \exp \left\{-\frac{1}{2}\left[\left(\boldsymbol{x}_{1}-\boldsymbol{\mu}_{1}^{(t)}\right)^{\mathrm{T}} \boldsymbol{P}_{1}^{(t)^{-1}}\left(\boldsymbol{x}_{1}-\boldsymbol{\mu}_{1}^{(t)}\right)+d^{(t)} \sum_{k=1}^{N}\left(\boldsymbol{z}_{k}-\boldsymbol{\mu}_{\boldsymbol{\Gamma}}^{(t)} \boldsymbol{x}_{k}\right)^{\mathrm{T}} \boldsymbol{D}^{(t)^{-1}}\left(\boldsymbol{z}_{k}-\boldsymbol{\mu}_{\boldsymbol{\Gamma}}^{(t)} \boldsymbol{x}_{k}\right)+\left(N_{s}+\right.\right.\right. \\
& \left.\left.\left.N_{o}\right) \sum_{k=1}^{N} \boldsymbol{x}_{k}^{\mathrm{T}} \boldsymbol{\Pi}^{(t)} \boldsymbol{x}_{k}\right]\right\}
\end{aligned}
$$

VBM step:

$$
q_{\boldsymbol{\Gamma} \boldsymbol{\Sigma}}^{(t+1)}(\boldsymbol{\gamma}, \boldsymbol{\sigma})=\operatorname{MN}\left(\boldsymbol{\mu}_{\boldsymbol{\Gamma}}^{(t+1)}, \boldsymbol{\Sigma}, \boldsymbol{\Pi}^{(t+1)}\right) \operatorname{IW}\left(d^{(t+1)}, \boldsymbol{D}^{(t+1)}\right)
$$

where

$$
\begin{aligned}
& \boldsymbol{\Pi}^{(t+1)}=\left[\boldsymbol{\Pi}^{-1}+\sum_{k=1}^{N} \mathrm{E}_{\boldsymbol{X}_{k} \mid \boldsymbol{Y}_{1: N}}\left(\boldsymbol{x}_{k} \boldsymbol{x}_{k}^{\mathrm{T}}\right)\right]^{-1}, \\
& \boldsymbol{\mu}_{\boldsymbol{\Gamma}}^{(t+1)}=\left[\boldsymbol{\mu}_{\boldsymbol{\Gamma}} \boldsymbol{\Pi}^{-1}+\sum_{k=1}^{N} \mathrm{E}_{\boldsymbol{X}_{k: k+1} \mid \boldsymbol{Y}_{1: N}}\left(\mathbf{z}_{k} \boldsymbol{x}_{k}^{\mathrm{T}}\right)\right] \boldsymbol{\Pi}^{(t+1)}, \\
& d^{(t+1)}=d+N \text {, } \\
& \boldsymbol{D}^{(t+1)}=\boldsymbol{D}+\sum_{k=1}^{N} \mathrm{E}_{\boldsymbol{X}_{k+1} \mid \boldsymbol{Y}_{1: N}}\left(\mathbf{z}_{k} \mathbf{z}_{k}^{\mathrm{T}}\right)+\boldsymbol{\mu}_{\boldsymbol{\Gamma}} \boldsymbol{\Pi}^{-1} \boldsymbol{\mu}_{\boldsymbol{\Gamma}}^{\mathrm{T}}-\boldsymbol{\mu}_{\boldsymbol{\Gamma}}^{(t+1)} \boldsymbol{\Pi}^{(t+1)^{-1}} \boldsymbol{\mu}_{\boldsymbol{\Gamma}}^{(t+1)^{\mathrm{T}}} \text {. }
\end{aligned}
$$

VBP step:

$$
\pi_{\boldsymbol{X}_{1}}^{(t+1)}\left(\boldsymbol{x}_{1}\right)=N\left(\boldsymbol{\mu}_{1}^{(t+1)}, \boldsymbol{P}_{1}^{(t+1)}\right)
$$


with $\boldsymbol{\mu}_{1}^{(t+1)}=\mathrm{E}_{\boldsymbol{X}_{1} \mid \boldsymbol{Y}_{1: N}}\left(\boldsymbol{x}_{1}\right)$ and $\boldsymbol{P}_{1}^{(t+1)}=\mathrm{E}_{\boldsymbol{X}_{k} \mid \boldsymbol{Y}_{1: N}}\left[\left(\boldsymbol{x}_{k}-\boldsymbol{\mu}_{1}^{(t+1)}\right)\left(\boldsymbol{x}_{k}-\boldsymbol{\mu}_{1}^{(t+1)}\right)^{\mathrm{T}}\right]$.

where $Z_{\boldsymbol{X}_{1: N+1}}$ is the normalizing constant. Moreover, each step increases $\mathrm{F}\left[q_{\boldsymbol{\Theta}}(\boldsymbol{\theta}), q_{\boldsymbol{X}_{1: N+1}}\left(\boldsymbol{x}_{1: N+1}\right)\right]$ monotonically with the sequence converging to a local maximum.

The proof of Theorem 2 is presented in Appendix B.

As stated in Section 2, the uncertainty would be underestimated if the priors are updated in each iteration; so, we choose not to update the prior of $\boldsymbol{\Theta}$ but only the prior of $\boldsymbol{X}_{1}$ to alleviate the underestimation. As shown in Eqn. (20), the estimation of the parameter matrix $\boldsymbol{\Theta}$ depends on all measurements. Updating its prior is equivalent to repeatedly using these measurements, i.e. artificially lengthening the data set, thus the uncertainty underestimation is expected. By choosing not to update the prior of $\boldsymbol{\Theta}$, its posterior will not be strongly influenced by the prior, and a more accurate estimate of the variance can be obtained. As for the prior updating of $\boldsymbol{X}_{1}$, it is because the uncertainty in the parameter $\boldsymbol{\Theta}$ are more concerned, and $\boldsymbol{X}_{1}$ has a trivial influence on that. The results of our implementation further validate our approach.

Because the VB algorithm is statistically consistent only if the starting distribution of the parameter matrix $\boldsymbol{\Theta}$ is sufficiently close to the true solution [25], the initial selection of the mean value is critical for its performance. In this paper, the prior distribution of $\boldsymbol{\Theta}$ is selected with a mean identified by the SSI algorithm [7] and a large variance to make this prior sufficiently flat. As a method of moments, the SSI algorithm usually gives good point estimates of modal parameters. Thus, we presume that modal parameters identified by the SSI algorithm are sufficiently close to the true ones to make the VB algorithm statistically consistent.

In order to iteratively increase the free energy function $\mathrm{F}\left[q_{\boldsymbol{\Theta}}(\boldsymbol{\theta}), q_{\boldsymbol{X}_{1: N+1}}\left(\boldsymbol{x}_{1: N+1}\right)\right]$, the expectation quantities in the VBM step must be evaluated. However, due to the special structure of $q_{\boldsymbol{X}_{1: N+1}}\left(\boldsymbol{x}_{1: N+1}\right)$, the standard Kalman smoother is not directly applicable, and a transformation of the SSM in Section 3 is needed in advance. This is achieved by the following lemma [30]:

Lemma 4.1. Considering the posterior distribution $q_{X_{1: N+1}}^{(t+1)}\left(\boldsymbol{x}_{1: N+1}\right)$ in Eqn. (19), the expectation terms in Eqn. (20) are calculated as follows:

$$
\begin{aligned}
& \mathrm{E}_{\boldsymbol{X}_{k} \mid \boldsymbol{Y}_{1: N}}\left(\boldsymbol{x}_{k} \boldsymbol{x}_{k}^{\mathrm{T}}\right)=\boldsymbol{P}_{k \mid N}+\boldsymbol{x}_{k \mid N} \boldsymbol{x}_{k \mid N}^{\mathrm{T}} \\
& \mathrm{E}_{\boldsymbol{X}_{k: k+1} \mid \boldsymbol{Y}_{1: N}}\left(\boldsymbol{z}_{k} \boldsymbol{x}_{k}^{\mathrm{T}}\right)=\left[\begin{array}{c}
\boldsymbol{P}_{k+1, k \mid N}+\boldsymbol{x}_{k+1 \mid N} \boldsymbol{x}_{k \mid N}^{\mathrm{T}} \\
\boldsymbol{y}_{k} \boldsymbol{x}_{k \mid N}^{\mathrm{T}}
\end{array}\right] \\
& \mathrm{E}_{\boldsymbol{X}_{k+1} \mid \boldsymbol{Y}_{1: N}}\left(\boldsymbol{z}_{k} \boldsymbol{z}_{k}^{\mathrm{T}}\right)=\left[\begin{array}{cc}
\boldsymbol{P}_{k+1 \mid N}+\boldsymbol{x}_{k+1 \mid N} \boldsymbol{x}_{k+1 \mid N}^{\mathrm{T}} & \boldsymbol{x}_{k+1 \mid N} \boldsymbol{y}_{k}^{\mathrm{T}} \\
\boldsymbol{y}_{k} \boldsymbol{x}_{k+1 \mid N}^{\mathrm{T}} & \boldsymbol{y}_{k} \boldsymbol{y}_{k}^{\mathrm{T}}
\end{array}\right]
\end{aligned}
$$

where $\boldsymbol{x}_{k \mid N}, \boldsymbol{P}_{k \mid N}$ and $\boldsymbol{P}_{k+1, k \mid N}$ are calculated via the Kalman smoother [31]

$$
\begin{aligned}
& \boldsymbol{L}_{k}=\boldsymbol{P}_{k \mid k} \widetilde{\boldsymbol{A}}^{\mathrm{T}} \boldsymbol{P}_{k+1 \mid k} \\
& \boldsymbol{x}_{k \mid N}=\boldsymbol{x}_{k \mid k}+\boldsymbol{L}_{k}\left(\boldsymbol{x}_{k+1 \mid N}-\widetilde{\boldsymbol{A}} \boldsymbol{x}_{k \mid k}-\tilde{\boldsymbol{S}} \widetilde{\boldsymbol{R}}^{-1} \widetilde{\boldsymbol{y}}_{k}\right)
\end{aligned}
$$




$$
\begin{aligned}
& \boldsymbol{P}_{k \mid N}=\boldsymbol{P}_{k \mid k}+\boldsymbol{L}_{k}\left(\boldsymbol{P}_{k+1 \mid N}-\boldsymbol{P}_{k+1 \mid k}\right) \boldsymbol{L}_{k}^{\mathrm{T}} \\
& \boldsymbol{P}_{k+1, k \mid N}=\boldsymbol{P}_{k+1 \mid k+1} \boldsymbol{L}_{k}^{\mathrm{T}}
\end{aligned}
$$

for $k=N, \ldots, 1$, and matrices $\widetilde{\boldsymbol{A}}, \widetilde{\boldsymbol{C}}, \widetilde{\boldsymbol{Q}}, \widetilde{\boldsymbol{R}}, \tilde{\boldsymbol{S}}$ and vector $\widetilde{\boldsymbol{y}}_{k}$ are computed as follows: First, make the following definitions and the Cholesky decomposition

$$
\left[\begin{array}{l}
\overline{\boldsymbol{A}} \\
\overline{\boldsymbol{C}}
\end{array}\right]=\boldsymbol{\mu}_{\boldsymbol{\Gamma}}^{(t)},\left[\begin{array}{cc}
\overline{\boldsymbol{Q}} & \overline{\boldsymbol{S}} \\
\overline{\boldsymbol{S}}^{\mathrm{T}} & \overline{\boldsymbol{R}}
\end{array}\right]=\frac{\boldsymbol{D}^{(t)}}{d-N_{s}-N_{o}-1},\left(N_{s}+N_{o}\right) \boldsymbol{\Pi}^{(t)}=\boldsymbol{U}^{\mathrm{T}} \boldsymbol{U}
$$

Then,

$$
\widetilde{\boldsymbol{y}}_{k}=\left[\begin{array}{c}
\boldsymbol{y}_{k} \\
\mathbf{0}_{N_{s}}
\end{array}\right], \widetilde{\boldsymbol{C}}=\left[\begin{array}{l}
\overline{\boldsymbol{C}} \\
\boldsymbol{U}
\end{array}\right], \widetilde{\boldsymbol{R}}=\left[\begin{array}{cc}
\overline{\boldsymbol{R}} & \mathbf{0}_{N_{d} \times N_{s}} \\
\mathbf{0}_{N_{s} \times N_{d}} & \boldsymbol{I}_{N_{s}}
\end{array}\right], \widetilde{\boldsymbol{A}}=\overline{\boldsymbol{A}}-\overline{\boldsymbol{S}} \overline{\boldsymbol{R}}^{-1} \overline{\boldsymbol{C}}, \widetilde{\boldsymbol{Q}}=\overline{\boldsymbol{Q}}-\overline{\boldsymbol{S}} \overline{\boldsymbol{R}}^{-1} \overline{\boldsymbol{S}}^{T}
$$

The quantities $\boldsymbol{x}_{k \mid k}, \boldsymbol{P}_{k \mid k}$ and $\boldsymbol{P}_{k+1 \mid k}$ appearing in Eqns. (25)-(28) are pre-calculated by the Kalman filter [31]

$$
\begin{aligned}
& \boldsymbol{x}_{k+1 \mid k}=\widetilde{\boldsymbol{A}} \boldsymbol{x}_{k \mid k}+\tilde{\boldsymbol{S}} \widetilde{\boldsymbol{R}}^{-1} \widetilde{\boldsymbol{y}}_{k} \\
& \boldsymbol{P}_{k+1 \mid k}=\widetilde{\boldsymbol{Q}}+\widetilde{\boldsymbol{A}} \boldsymbol{P}_{k \mid k} \widetilde{\boldsymbol{A}}^{\mathrm{T}} \\
& \boldsymbol{K}_{k+1}=\boldsymbol{P}_{k+1 \mid k} \widetilde{\boldsymbol{C}}^{\mathrm{T}}\left[\widetilde{\boldsymbol{R}}+\widetilde{\boldsymbol{C}} \boldsymbol{P}_{k+1 \mid k} \widetilde{\boldsymbol{C}}^{\mathrm{T}}\right]^{-1} \\
& \boldsymbol{x}_{k+1 \mid k+1}=\boldsymbol{x}_{k+1 \mid k}+\boldsymbol{K}_{k+1}\left(\widetilde{\boldsymbol{y}}_{k+1}-\widetilde{\boldsymbol{C}} \boldsymbol{x}_{k+1 \mid k}\right) \\
& \boldsymbol{P}_{k+1 \mid k+1}=\boldsymbol{P}_{k+1 \mid k}-\boldsymbol{K}_{k+1} \widetilde{\boldsymbol{C}} \boldsymbol{P}_{k+1 \mid k}
\end{aligned}
$$

for $k=1, \ldots, N$, and the sequence is initialized by $\boldsymbol{x}_{1 \mid 1}=\boldsymbol{\mu}_{1}^{(t)}$ and $\boldsymbol{P}_{1 \mid 1}=\boldsymbol{P}_{1}^{(t)}$.

\section{Implementation and Postprocessing}

5.1 Free Energy Evaluation

Theoretically, the free energy $\mathrm{F}\left[q_{\boldsymbol{\theta}}(\boldsymbol{\theta}), q_{X_{1: N+1}}\left(\boldsymbol{x}_{1: N+1}\right)\right]$ increases monotonically and converges to a local maximum. However, in implementation, one has to evaluate the free energy in order to confirm its convergence. Due to the high dimension of the integration, direct evaluation of the free energy is difficult. Here, by inspecting the VB-EMP steps, we develop a simplified procedure as stated in Lemma 5.1.

Lemma 5.1 Using the augmented stochastic state space model in Eqn. (35), the free energy is calculated after the VBM step as

$$
\mathrm{F}\left[q_{\boldsymbol{\theta}}(\boldsymbol{\theta}), q_{\boldsymbol{X}_{1: N+1}}\left(\boldsymbol{x}_{1: N+1}\right)\right]=1+\frac{N}{2} \ln |\widetilde{\boldsymbol{Q}}|+\frac{N}{2} \ln |\widetilde{\boldsymbol{R}}|+\frac{1}{2} \sum_{k=1}^{N} \operatorname{tr}\left\{\left[\begin{array}{ll}
\boldsymbol{E}_{k} & \boldsymbol{F}_{k} \\
\boldsymbol{F}_{k}^{\mathrm{T}} & \boldsymbol{G}_{k}
\end{array}\right]\left[\begin{array}{cc}
\widetilde{\boldsymbol{Q}} & \mathbf{0}_{N_{S} \times\left(N_{s}+N_{o}\right)} \\
\mathbf{0}_{\left(N_{S}+N_{o}\right) \times N_{S}} & \widetilde{\boldsymbol{R}}
\end{array}\right]^{-1}\right\}
$$

where

$$
\begin{gathered}
\boldsymbol{E}_{k}=\mathrm{E}_{\boldsymbol{X}_{k+1} \mid \boldsymbol{Y}_{1: N}}\left(\boldsymbol{x}_{k+1} \boldsymbol{x}_{k+1}^{\mathrm{T}}\right)-\mathrm{E}_{\boldsymbol{X}_{k: k+1} \mid \boldsymbol{Y}_{1: N}}\left(\boldsymbol{x}_{k+1} \boldsymbol{x}_{k}^{\mathrm{T}}\right) \widetilde{\boldsymbol{A}}^{\mathrm{T}}-\widetilde{\boldsymbol{A}} \mathrm{E}_{\boldsymbol{X}_{k: k+1} \mid \boldsymbol{Y}_{1: N}}\left(\boldsymbol{x}_{k} \boldsymbol{x}_{k+1}^{\mathrm{T}}\right)+\widetilde{\boldsymbol{A}} \mathrm{E}_{\boldsymbol{X}_{k} \mid \boldsymbol{Y}_{1: N}}\left(\boldsymbol{x}_{k} \boldsymbol{x}_{k}^{\mathrm{T}}\right) \widetilde{\boldsymbol{A}}^{\mathrm{T}} \\
\boldsymbol{F}_{k}=\mathrm{E}_{\boldsymbol{X}_{k+1} \mid \boldsymbol{Y}_{1: N}}\left(\boldsymbol{x}_{k+1}\right) \widetilde{\boldsymbol{y}}_{k}^{\mathrm{T}}-\mathrm{E}_{\boldsymbol{X}_{k: k+1} \mid \boldsymbol{Y}_{1: N}}\left(\boldsymbol{x}_{k+1} \boldsymbol{x}_{k}^{\mathrm{T}}\right) \widetilde{\boldsymbol{C}}^{\mathrm{T}}-\widetilde{\boldsymbol{A}} \mathrm{E}_{\boldsymbol{X}_{k} \mid \boldsymbol{Y}_{1: N}}\left(\boldsymbol{x}_{k}\right) \widetilde{\boldsymbol{y}}_{k}^{\mathrm{T}}+\widetilde{\boldsymbol{A}} \mathrm{E}_{\boldsymbol{X}_{k} \mid \boldsymbol{Y}_{1: N}}\left(\boldsymbol{x}_{k} \boldsymbol{x}_{k}^{\mathrm{T}}\right) \widetilde{\boldsymbol{C}}^{\mathrm{T}}
\end{gathered}
$$




$$
\boldsymbol{G}_{k}=\widetilde{\boldsymbol{y}}_{k} \widetilde{\boldsymbol{y}}_{k}^{\mathrm{T}}-\widetilde{\boldsymbol{y}}_{k} \mathrm{E}_{\boldsymbol{X}_{k} \mid \boldsymbol{Y}_{1: N}}\left(\boldsymbol{x}_{k}^{\mathrm{T}}\right) \widetilde{\boldsymbol{C}}^{\mathrm{T}}-\widetilde{\boldsymbol{C}} \mathrm{E}_{\boldsymbol{X}_{k} \mid \boldsymbol{Y}_{1: N}}\left(\boldsymbol{x}_{k}\right) \widetilde{\boldsymbol{y}}_{k}^{\mathrm{T}}+\widetilde{\boldsymbol{C}} \mathrm{E}_{\boldsymbol{X}_{k} \mid \boldsymbol{Y}_{1: N}}\left(\boldsymbol{x}_{k} \boldsymbol{x}_{k}^{\mathrm{T}}\right) \widetilde{\boldsymbol{C}}^{\mathrm{T}}
$$

All terms in the above expectations are solved as part of Lemma 4.1. The proof of the above lemma is shown in Appendix B.

After evaluating the free energy, we still need a criterion to determine whether the iteration is convergent or not. One typical convergence criterion used for applications such as this is a measure of the relative change in the quantity of interest between successive steps. Denote $\mathrm{F}^{(t)}$ as the free energy at the $t$-th iteration; the convergence criterion can then be described as:

$$
\frac{\mathrm{F}^{(t+1)}-\mathrm{F}^{(t)}}{\left(\mathrm{F}^{(t+1)}+\mathrm{F}^{(t)}\right) / 2} \leq \rho
$$

The averaging in the denominator is used to increase the stability of the condition. A typical value for $\rho$ in such a condition is $10^{-5} \sim 10^{-4}$. It is well-known that the convergence of EM-like algorithms may be infinitely long [32], but since we start with relatively good initial parameters obtained by the SSI algorithm, the stopping criterion in Eqn. (37) usually yields a result that is sufficiently close to the true solution in practical applications.

\subsection{Robust implementation}

Theorem 2 provides an iterative way to update the posterior distributions of the latent variables and parameters, but naïve implementations directly following the updating steps may suffer a serious numerical error. Since the matrices $\boldsymbol{P}_{k \mid k}, \boldsymbol{P}_{k+1 \mid k}, \boldsymbol{P}_{k+1 \mid N}, D^{(t+1)}$ and $\boldsymbol{\Pi}^{(t+1)}$ must be kept symmetric and positive semidefinite at all iterations, a robust implementation of the scheme is essential. The square-root filtering strategy developed by Gibon and Ninness [33] can be applied in calculating the first three covariance matrices. For computing $\boldsymbol{\mu}_{\boldsymbol{\Gamma}}^{(t+1)}, \boldsymbol{D}^{(t+1)}$ and $\boldsymbol{\Pi}^{(t+1)}$, the Cholesky decomposition

$$
\left[\begin{array}{cc}
\boldsymbol{H}^{(t)} & \boldsymbol{J}^{(t)^{\mathrm{T}}} \\
\boldsymbol{J}^{(t)} & \boldsymbol{K}^{(t)}
\end{array}\right]=\left[\begin{array}{cc}
\boldsymbol{L}_{11} & \mathbf{0} \\
\boldsymbol{L}_{21} & \boldsymbol{L}_{22}
\end{array}\right]\left[\begin{array}{cc}
\boldsymbol{L}_{11} & \mathbf{0} \\
\boldsymbol{L}_{21} & \boldsymbol{L}_{22}
\end{array}\right]^{\mathrm{T}}=\left[\begin{array}{cc}
\boldsymbol{L}_{11} \boldsymbol{L}_{11}^{\mathrm{T}} & \boldsymbol{L}_{11} \boldsymbol{L}_{21}^{\mathrm{T}} \\
\boldsymbol{L}_{21} \boldsymbol{L}_{11}^{\mathrm{T}} & \boldsymbol{L}_{21} \boldsymbol{L}_{21}^{\mathrm{T}}+\boldsymbol{L}_{22} \boldsymbol{L}_{22}^{\mathrm{T}}
\end{array}\right]
$$

is employed, where all matrices are portioned conformably to the left-hand side and

$$
\begin{gathered}
\boldsymbol{H}^{(t)}=\boldsymbol{\Pi}^{-1}+\sum_{k=1}^{N} \mathrm{E}_{\boldsymbol{X}_{k} \mid \boldsymbol{Y}_{1: N}}\left(\boldsymbol{x}_{k} \boldsymbol{x}_{k}^{\mathrm{T}}\right) \\
\boldsymbol{J}^{(t)}=\boldsymbol{\mu}_{\boldsymbol{\Gamma}} \boldsymbol{\Pi}^{-1}+\sum_{k=1}^{N} \mathrm{E}_{\boldsymbol{X}_{k: k+1} \mid \boldsymbol{Y}_{1: N}}\left(\mathbf{z}_{k} \boldsymbol{x}_{k}^{\mathrm{T}}\right) \\
\boldsymbol{K}^{(t)}=\boldsymbol{D}+\sum_{k=1}^{N} \mathrm{E}_{\boldsymbol{X}_{k+1} \mid \boldsymbol{Y}_{1: N}}\left(\mathbf{z}_{k} \boldsymbol{z}_{k}^{\mathrm{T}}\right)+\boldsymbol{\mu}_{\boldsymbol{\Gamma}} \boldsymbol{\Pi}^{-1} \boldsymbol{\mu}_{\boldsymbol{\Gamma}}^{\mathrm{T}}
\end{gathered}
$$

By equating the sub-matrices, we have

$$
\begin{aligned}
& \boldsymbol{\Pi}^{(t+1)^{-1}}=\boldsymbol{L}_{11} \boldsymbol{L}_{11}^{\mathrm{T}} \\
& \boldsymbol{\mu}_{\Gamma}^{(t+1)}=\boldsymbol{L}_{21} \boldsymbol{L}_{11}^{\mathrm{T}}\left(\boldsymbol{L}_{11} \boldsymbol{L}_{11}^{\mathrm{T}}\right)^{-1}=\boldsymbol{L}_{21} \boldsymbol{L}_{11}^{-1} \\
& \boldsymbol{D}^{(t+1)}=\boldsymbol{L}_{21} \boldsymbol{L}_{21}^{\mathrm{T}}+\boldsymbol{L}_{22} \boldsymbol{L}_{22}^{\mathrm{T}}-\boldsymbol{L}_{21} \boldsymbol{L}_{11}^{-1} \boldsymbol{L}_{11} \boldsymbol{L}_{11}^{\mathrm{T}}\left(\boldsymbol{L}_{21} \boldsymbol{L}_{11}^{-1}\right)^{\mathrm{T}}=\boldsymbol{L}_{22} \boldsymbol{L}_{22}^{\mathrm{T}}
\end{aligned}
$$

Eqns. (39) and (41) guarantee the symmetry and positive semi-definiteness of the covariance matrices, and Eqn. (40) provides an efficient way to compute $\boldsymbol{\mu}_{\Gamma}^{(t+1)}$. 
5.3 Distributions of modal parameters

Although the posterior distribution $p_{\Gamma \Sigma}(\boldsymbol{\gamma}, \boldsymbol{\sigma})$ can be obtained using the robust implementation of Theorem 2, the distributions of modal parameters cannot directly be extracted. As described in Lemma A.1 in Appendix A, the marginal distribution of $\boldsymbol{\Gamma}$ will be a matrix variate- $t$ marginal distribution. However, there is no closed-form solution for the distribution of the modal parameters because of the eigenvalue decomposition involved in transforming $\boldsymbol{\Gamma}$ to the modal parameters, as shown in Eqn. (13). The perturbation method [13] and asymptotic analysis [34] have been proposed to approximate these distributions. Here, we choose the first-order Taylor series expansion at the posterior mean value $\boldsymbol{\mu}_{\boldsymbol{\Gamma}}^{*}$ to approximate the posterior joint distribution of modal parameters. This approximation is expected to provide a good estimation, because this joint distribution is generally unimodal and there are small coefficients of variation for modal parameters. Since the modal frequencies and damping ratios are always positive for stable structures, the first-order Taylor expansion is operated on their logrithms

$$
\left[\begin{array}{c}
\ln f_{i}(\boldsymbol{\Gamma}) \\
\ln \xi_{i}(\boldsymbol{\Gamma}) \\
\boldsymbol{\phi}_{i}(\boldsymbol{\Gamma})
\end{array}\right] \approx\left[\begin{array}{c}
\ln f_{i}\left(\boldsymbol{\mu}_{\Gamma}^{*}\right) \\
\ln \xi_{i}\left(\boldsymbol{\mu}_{\Gamma}^{*}\right) \\
\boldsymbol{\phi}_{i}\left(\boldsymbol{\mu}_{\boldsymbol{\Gamma}}^{*}\right)
\end{array}\right]+\left[\left.\begin{array}{c}
\frac{\partial \ln f_{i}(\boldsymbol{\Gamma})}{\partial \operatorname{vec}(\boldsymbol{\Gamma})} \\
\frac{\partial \ln \xi_{i}(\boldsymbol{\Gamma})}{\partial \operatorname{vec}(\boldsymbol{\Gamma})} \\
\frac{\partial \boldsymbol{\phi}_{i}(\boldsymbol{\Gamma})}{\partial \operatorname{vec}(\boldsymbol{\Gamma})}
\end{array}\right|_{\boldsymbol{\Gamma}=\boldsymbol{\mu}_{\boldsymbol{\Gamma}}^{*}}\left[\operatorname{vec}(\boldsymbol{\Gamma})-\operatorname{vec}\left(\boldsymbol{\mu}_{\boldsymbol{\Gamma}}^{*}\right)\right]\right.
$$

where 'vec(')' means stacking the columns of the matrix into a column vector, and the partial derivatives with respect to this vector is defined to be a row vector here.

Each element in the Jacobian matrix in Eqn. (42) can be calculated using the chain rule as follows:

$$
\begin{aligned}
& \frac{\partial \ln f_{i}(\Gamma)}{\partial a_{m n}}=\frac{\partial \ln f_{i}(\Gamma)}{\partial f_{i}(\Gamma)} \frac{\partial f_{i}\left(\lambda_{c i}\right)}{\partial \lambda_{c i}} \frac{\partial \lambda_{c i}}{\partial \lambda_{i}} \frac{\partial \lambda_{i}}{\partial a_{m n}}=\frac{1}{f_{i}}\left[\frac{\operatorname{Re}\left(\lambda_{c i}\right)}{2 \pi\left|\lambda_{c i}\right|} \quad \frac{\operatorname{Im}\left(\lambda_{c i}\right)}{2 \pi \mid \lambda_{c i} i}\right]\left[\begin{array}{cc}
\frac{\operatorname{Re}\left(\lambda_{i}\right)}{T_{s}\left|\lambda_{i}\right|^{2}} & \frac{\operatorname{Im}\left(\lambda_{i}\right)}{T_{s}\left|\lambda_{i}\right|^{2}} \\
-\frac{\operatorname{Im}\left(\lambda_{i}\right)}{T_{s}\left|\lambda_{i}\right|^{2}} & \frac{\operatorname{Re}\left(\lambda_{i}\right)}{T_{s}\left|\lambda_{i}\right|^{2}}
\end{array}\right]\left[\begin{array}{c}
\frac{\partial \operatorname{Re}\left(\lambda_{i}\right)}{\partial a_{m n}} \\
\frac{\partial \operatorname{Im}\left(\lambda_{i}\right)}{\partial a_{m n}}
\end{array}\right] \\
& \frac{\partial \ln \xi_{i}(\Gamma)}{\partial a_{m n}}=\frac{\partial \ln \xi_{i}(\Gamma)}{\partial \xi_{i}(\Gamma)} \frac{\partial \xi_{i}\left(\lambda_{c i}\right)}{\partial \lambda_{c i}} \frac{\partial \lambda_{c i}}{\partial \lambda_{i}} \frac{\partial \lambda_{i}}{\partial a_{m n}}=\frac{1}{\xi_{i}}\left[-\frac{\operatorname{Im}\left(\lambda_{c i}\right)^{2}}{\left|\lambda_{c i}\right|^{3}} \quad \frac{\operatorname{Re}\left(\lambda_{c i}\right) \operatorname{Im}\left(\lambda_{c i}\right)}{\left|\lambda_{c i}\right|^{3}}\right]\left[\begin{array}{cc}
\frac{\operatorname{Re}\left(\lambda_{i}\right)}{T_{s}\left|\lambda_{i}\right|^{2}} & \frac{\operatorname{Im}\left(\lambda_{i}\right)}{T_{s}\left|\lambda_{i}\right|^{2}} \\
-\frac{\operatorname{Im}\left(\lambda_{i}\right)}{T_{s}\left|\lambda_{i}\right|^{2}} & \frac{\operatorname{Re}\left(\lambda_{i}\right)}{T_{s}\left|\lambda_{i}\right|^{2}}
\end{array}\right]\left[\begin{array}{l}
\frac{\partial \operatorname{Re}\left(\lambda_{i}\right)}{\partial a_{m n}} \\
\frac{\partial \operatorname{Im}\left(\lambda_{i}\right)}{\partial a_{m n}}
\end{array}\right] \\
& \frac{\partial \boldsymbol{\phi}_{i}(\boldsymbol{\Gamma})}{\partial a_{m n}}=\boldsymbol{C}\left[\frac{\partial \operatorname{Re}\left(\boldsymbol{\psi}_{i}\right)}{\partial a_{m n}} \frac{\partial \operatorname{Im}\left(\boldsymbol{\psi}_{i}\right)}{\partial a_{m n}}\right] \boldsymbol{P}_{2} \boldsymbol{z}+\boldsymbol{C} \boldsymbol{\varphi}_{i} \boldsymbol{P}_{2} \frac{\partial \boldsymbol{z}}{\partial a_{m n}} \\
& \frac{\partial \ln f_{i}(\boldsymbol{\Gamma})}{\partial c_{m n}}=\frac{\partial \ln \xi_{i}(\boldsymbol{\Gamma})}{\partial c_{m n}}=0, \frac{\partial \boldsymbol{\phi}_{i}(\boldsymbol{\Gamma})}{\partial c_{m n}}=\boldsymbol{e}_{m} \boldsymbol{e}_{n}^{\mathrm{T}} \boldsymbol{\varphi}_{i} \boldsymbol{P}_{2} \boldsymbol{z}+\boldsymbol{C} \boldsymbol{\varphi}_{i} \boldsymbol{P}_{2} \frac{\partial \mathbf{z}}{\partial c_{m n}}
\end{aligned}
$$

Computation of the partial derivatives of eigenvalues and eigenvectors is described in Appendix C. Similarly, we can compute the first-order Taylor expansion for all identified modes and then formulate them as a linear equation

$$
\boldsymbol{\Xi}(\boldsymbol{\Gamma}) \approx \boldsymbol{\Xi}\left(\boldsymbol{\mu}_{\Gamma}^{*}\right)+\left.\boldsymbol{J}(\boldsymbol{\Gamma})\right|_{\Gamma=\mu_{\Gamma}^{*}}\left[\operatorname{vec}(\boldsymbol{\Gamma})-\operatorname{vec}\left(\boldsymbol{\mu}_{\Gamma}^{*}\right)\right]
$$

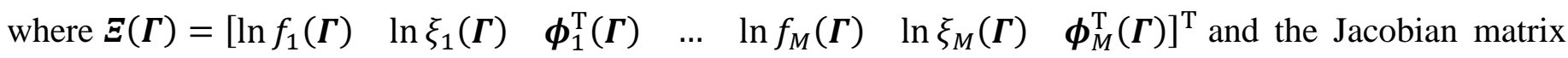
$\boldsymbol{J}(\boldsymbol{\Gamma})=\left[\begin{array}{llll}\boldsymbol{J}_{1}^{\mathrm{T}}(\boldsymbol{\Gamma}) & \ldots & \boldsymbol{J}_{M}^{\mathrm{T}}(\boldsymbol{\Gamma})\end{array}\right]^{\mathrm{T}}$ with each submatrix being the form shown in Eqn. (42). 
According to Lemma A.2, the matrix variate- $t$ distribution converges to a matrix normal distribution when the degree of freedom tends to infinity. In our case, the degree of freedom $d$ will be huge when the sequence of data is long, so that $\operatorname{vec}(\boldsymbol{\Gamma})$ is approximately multivariate normal distributed with mean $\operatorname{vec}\left(\boldsymbol{\mu}_{\boldsymbol{\Gamma}}^{*}\right)$ and covariance matrix $\boldsymbol{\Pi}^{*} \otimes \boldsymbol{D}^{*} /\left(d^{*}-N_{s}-N_{o}-1\right)$, where $\boldsymbol{\mu}_{\boldsymbol{\Gamma}}^{*}, \boldsymbol{\Pi}^{*}, \boldsymbol{D}^{*}$ and $d^{*}$ are the parameters upon convergence, and ' $\otimes$ ' stands for the Kronecker product. Therefore, the parameter $\boldsymbol{\Xi}(\boldsymbol{\Gamma})$ approximately follows a multivariate normal distribution with mean $\boldsymbol{\Xi}\left(\boldsymbol{\mu}_{\boldsymbol{\Gamma}}^{*}\right)$ and covariance matrix $\boldsymbol{J}\left(\boldsymbol{\mu}_{\boldsymbol{\Gamma}}^{*}\right)\left(\boldsymbol{\Pi}^{*} \otimes \boldsymbol{D}^{*}\right) \boldsymbol{J}\left(\boldsymbol{\mu}_{\boldsymbol{\Gamma}}^{*}\right)^{\mathrm{T}} /$ $\left(d^{*}-N_{s}-N_{o}-1\right)$. Because the logrithms of the modal frequency and damping ratio follow normal distributions, the modal frequency and damping ratio have lognormal distributions. The posterior of mode shapes is approximated by a multivariate normal distribution.

\section{Empirical Study}

Having made a robust implementation of the VB algorithm, this section provides an empirical study of its performance via a mass-spring system and a real structure - the One Rincon Tower in San Francisco, CA. The numerical example is used to evaluate the consistency in uncertainty quantification as well as the convergence property of the algorithm, while the second example demonstrates its applicability of the method to large-scale structure identification. In addition to the VB algorithm, the Expectation-Maximization algorithm [17] and Gibbs sampling [31] are implemented for comparison, and all are initialized by the same parameters obtained from a prior SSI [7].

\subsection{Mass-Spring System}

The mass-spring system is an idealization of a real structure. By controlling the true values of the parameters in the model, it becomes possible to use such a model to compare the performance of different algorithms. We adopt the mass-spring model in Fig. 5, which has previously been used in by Cara et al. [35].

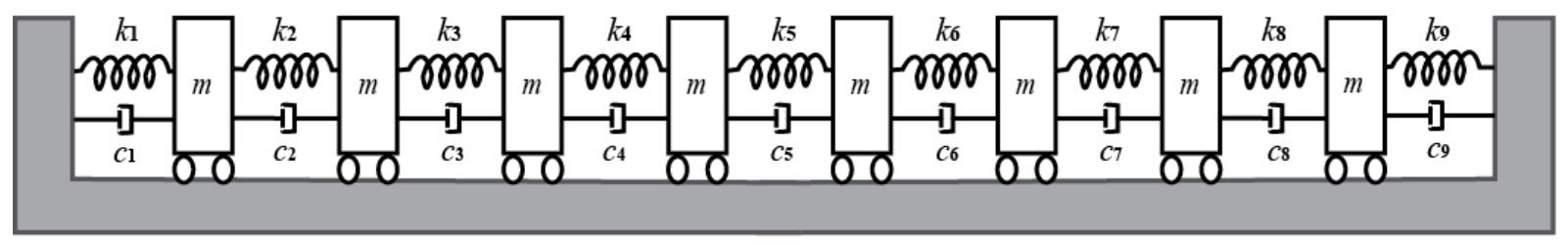

Fig. 5 Eight DOFs mass-spring system

The assumed model parameters are: $m=1, k_{i}=800 i$ for $i=1, \ldots, 9$, and Rayleigh damping with damping matrix $\boldsymbol{C}_{d}=0.68 \boldsymbol{M}+1.743 \times 10^{-4} \boldsymbol{K}$, where $\boldsymbol{M}$ and $\boldsymbol{K}$ are the mass and stiffness matrices, respectively. An identical band-limited, Gaussian white-noise forcing function is applied at each DOF. Acceleration responses at DOFs 2, 4, 6 and 8 are recorded with sampling frequency $25 \mathrm{~Hz}$ for 200 seconds. These "measured" responses are contaminated by an independent band-limited, Gaussian white-noise process with variance equal to $25 \%$ of the largest acceleration variance.

Table 1 lists the identified modal frequencies and damping ratios for the three methods. Note that the Expectation-Maximization (EM) algorithm provides only a point estimate. For VB and Gibbs sampling, the coefficient of variation (COV) or standard deviation (STD) of each estimate is provided. For Gibbs sampling, 1,800 samples are used after discarding burn-in samples. Comparing the point estimates (MLE or posterior 
means), all three methods are consistent, though slight biases exist. Among the three methods, EM seems to provide the best point estimates, while VB and Gibbs give similar performance. The estimated COVs of VB are always smaller than those of Gibbs sampler, by about 15\%. It is noted that the estimated COVs of the modal frequencies by both methods are extremely small.

Tab.1 Identified Modal Parameters using EM, VB and Gibbs Sampler

\begin{tabular}{|c|c|c|c|c|c|c|c|c|c|c|c|c|c|c|}
\hline \multirow{3}{*}{ Mode } & \multicolumn{5}{|c|}{ Frequencies $(\mathrm{Hz})$} & \multicolumn{9}{|c|}{ Damping Ratios (\%) } \\
\hline & \multirow{2}{*}{ True } & \multirow{2}{*}{ EM } & \multicolumn{2}{|c|}{$\mathrm{VB}$} & \multirow{2}{*}{$\begin{array}{l}\text { Gibbs } \\
\text { Mean }\end{array}$} & \multirow[b]{2}{*}{$\operatorname{COV}(\%)$} & \multirow{2}{*}{ True } & \multirow{2}{*}{ EM } & \multicolumn{3}{|c|}{ VB } & \multicolumn{3}{|c|}{ Gibbs } \\
\hline & & & Mean & $\operatorname{COV}(\%)$ & & & & & Mean & STD & $\operatorname{COV}(\%)$ & Mean & STD & $\operatorname{COV}(\%)$ \\
\hline 1 & 2.94 & 2.92 & 2.94 & 0.26 & 2.93 & 0.34 & 2.00 & 2.00 & 1.93 & 0.21 & 10.88 & 1.87 & 0.34 & 18.18 \\
\hline 2 & 5.87 & 5.88 & 5.87 & 0.20 & 5.88 & 0.21 & 1.24 & 1.16 & 1.30 & 0.18 & 13.84 & 1.29 & 0.21 & 16.28 \\
\hline 3 & 8.60 & 8.57 & 8.56 & 0.14 & 8.56 & 0.16 & 1.10 & 1.07 & 1.09 & 0.14 & 12.84 & 1.03 & 0.16 & 15.53 \\
\hline 4 & 11.19 & 11.21 & 11.22 & 0.11 & 11.20 & 0.13 & 1.09 & 0.79 & 0.78 & 0.10 & 12.82 & 0.84 & 0.13 & 15.47 \\
\hline 5 & 13.78 & 13.77 & 13.74 & 0.12 & 13.75 & 0.15 & 1.15 & 1.22 & 1.24 & 0.12 & 9.68 & 1.33 & 0.16 & 12.03 \\
\hline 6 & 16.52 & 16.54 & 16.54 & 0.11 & 16.55 & 0.13 & 1.23 & 1.07 & 1.20 & 0.11 & 9.17 & 1.12 & 0.13 & 11.61 \\
\hline 7 & 19.54 & 19.54 & 19.53 & 0.12 & 19.56 & 0.17 & 1.35 & 1.34 & 1.78 & 0.14 & 7.87 & 1.33 & 0.17 & 12.78 \\
\hline 8 & 23.12 & 23.09 & 23.08 & 0.11 & 23.06 & 0.14 & 1.50 & 1.56 & 1.77 & 0.13 & 7.34 & 1.44 & 0.15 & 10.42 \\
\hline
\end{tabular}
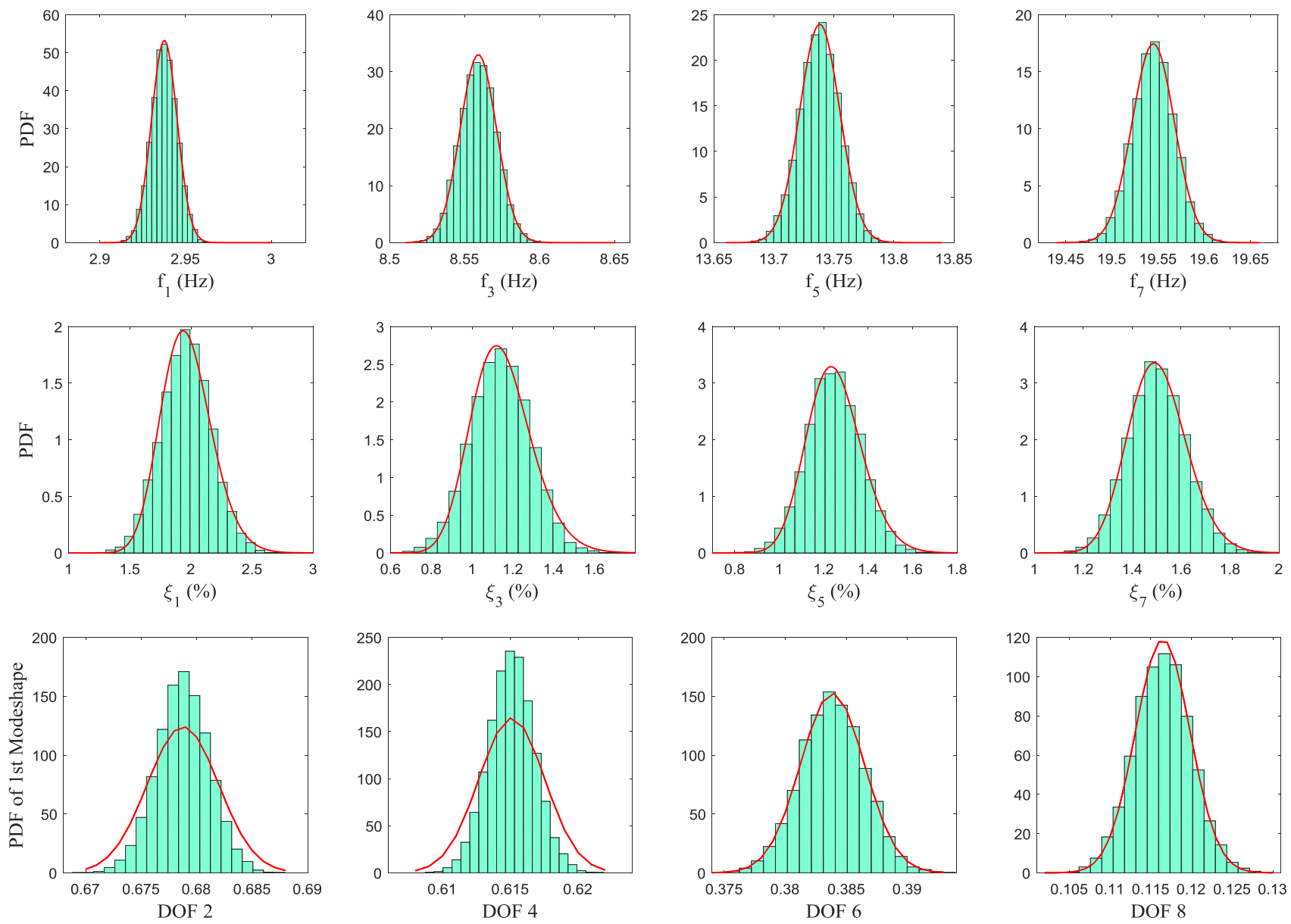

Fig. 6 First-order approximation of posterior distributions of modal parameters 
It is interesting to see that the estimated COVs of the frequencies are almost identical to the estimated standard deviations of the damping ratios for all physical modes. At this time we are unable to explain the reason for this phenomenon. Many previous studies have shown similar results, see, e.g., Refs [13, 18, 19], though they did not explicitly state this result.

In order to examine the accuracy of the posterior distributions of modal parameters obtained by the firstorder Taylor series expansion, 10,000 samples are generated from the posterior distribution of parameter $\boldsymbol{\Gamma}$ estimated by VB, as plotted in Fig. 6. The first-order approximation of the distribution of modal frequencies matches with the normalized frequency diagrams of the generated samples nearly perfectly. The approximation for the damping ratio is also good in spite of the larger COVs. For the mode shapes, discrepancies exist between the distributions obtained by first-order approximation and by sampling, in spite of the small COVs. This has to do with the normalization of the mode shapes in sampling - all mode shape samples are normalized to have unit length. As a result, the normalized frequency diagram does not correctly represent the true distribution of the mode shapes. Nevertheless, the mean values are in close agreement.

Comparison of cumulative distribution functions (CDFs) and empirical CDFs of the modal frequencies and damping ratios as obtained by VB and Gibbs sampler, respectively, are shown in Figs. 7 and 8, respectively. Again, we can see that the distributions estimated by VB show less dispersion than those obtained from the Gibbs sampler, though the two distributions are relatively close in most cases. Plots of the identified mode shapes, with the data for each DOF shown as a boxplot, are illustrated in Fig. 9. The same normalization method is used for the two sets of mode shapes. Both the VB and Gibbs sampler provide accurate estimates of the mode shapes, with the variations in identified mode shapes by VB being smaller than those estimated by the Gibbs sampler. In conclusion, the VB estimator is consistent, but tends to slightly under-estimate the uncertainty.
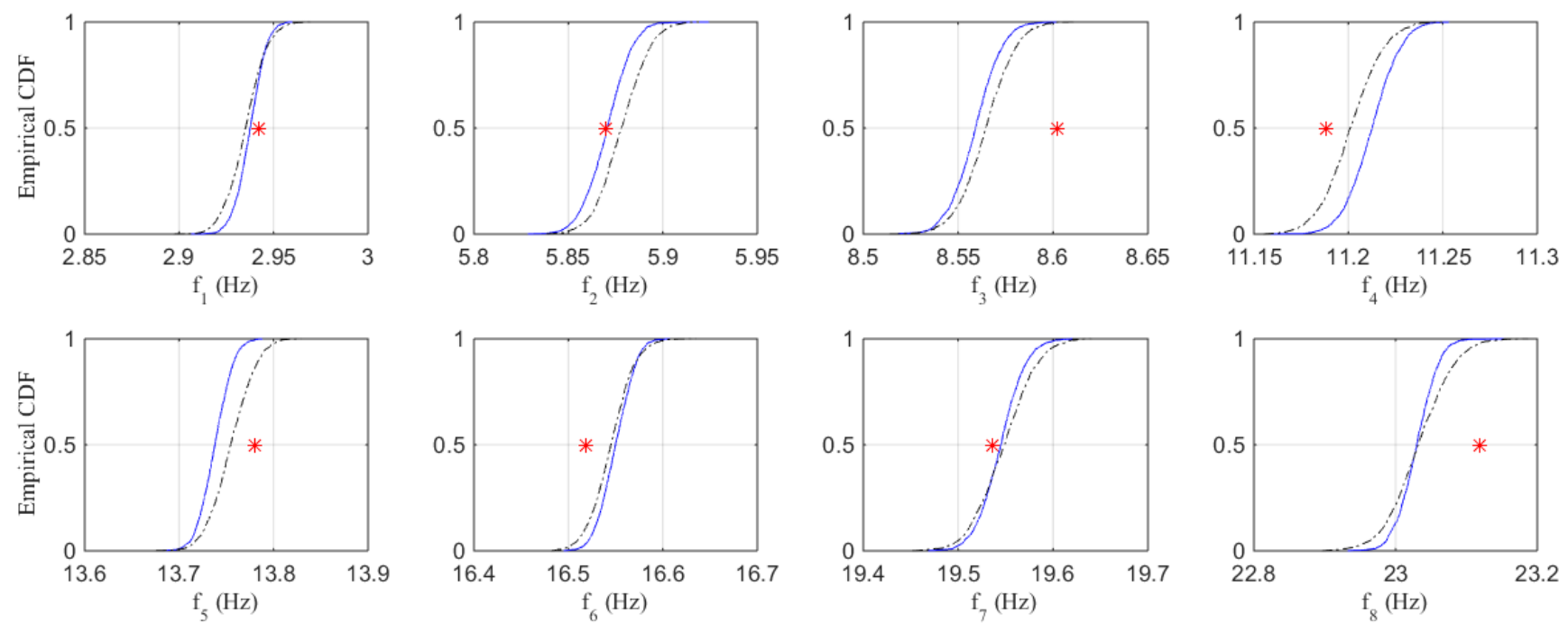

Fig. 7 Empirical CDF of identified modal frequencies Solid line: variational Bayes; Dash-dot line: Gibbs sampler; Star: true value 

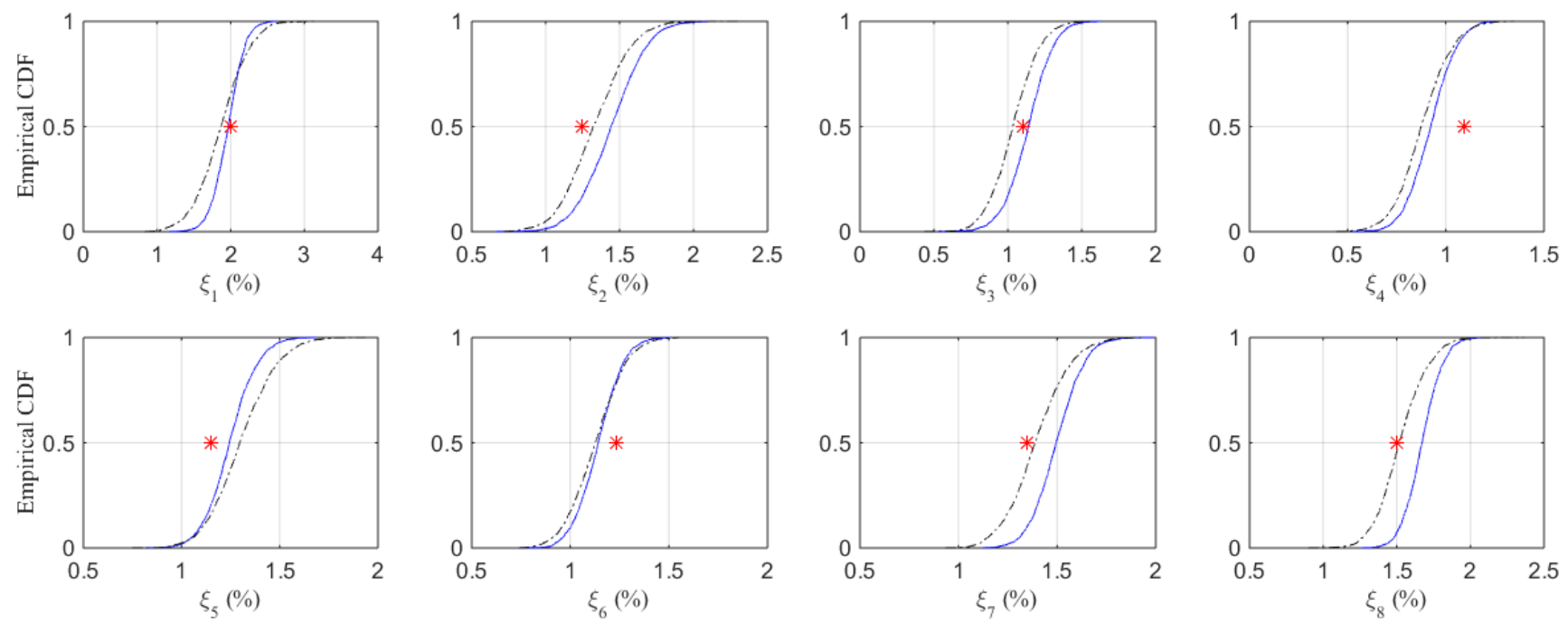

Fig. 8 Empirical CDF of identified damping ratios

Solid line: variational Bayes; Dash-dot line: Gibbs sampler; Star: true value
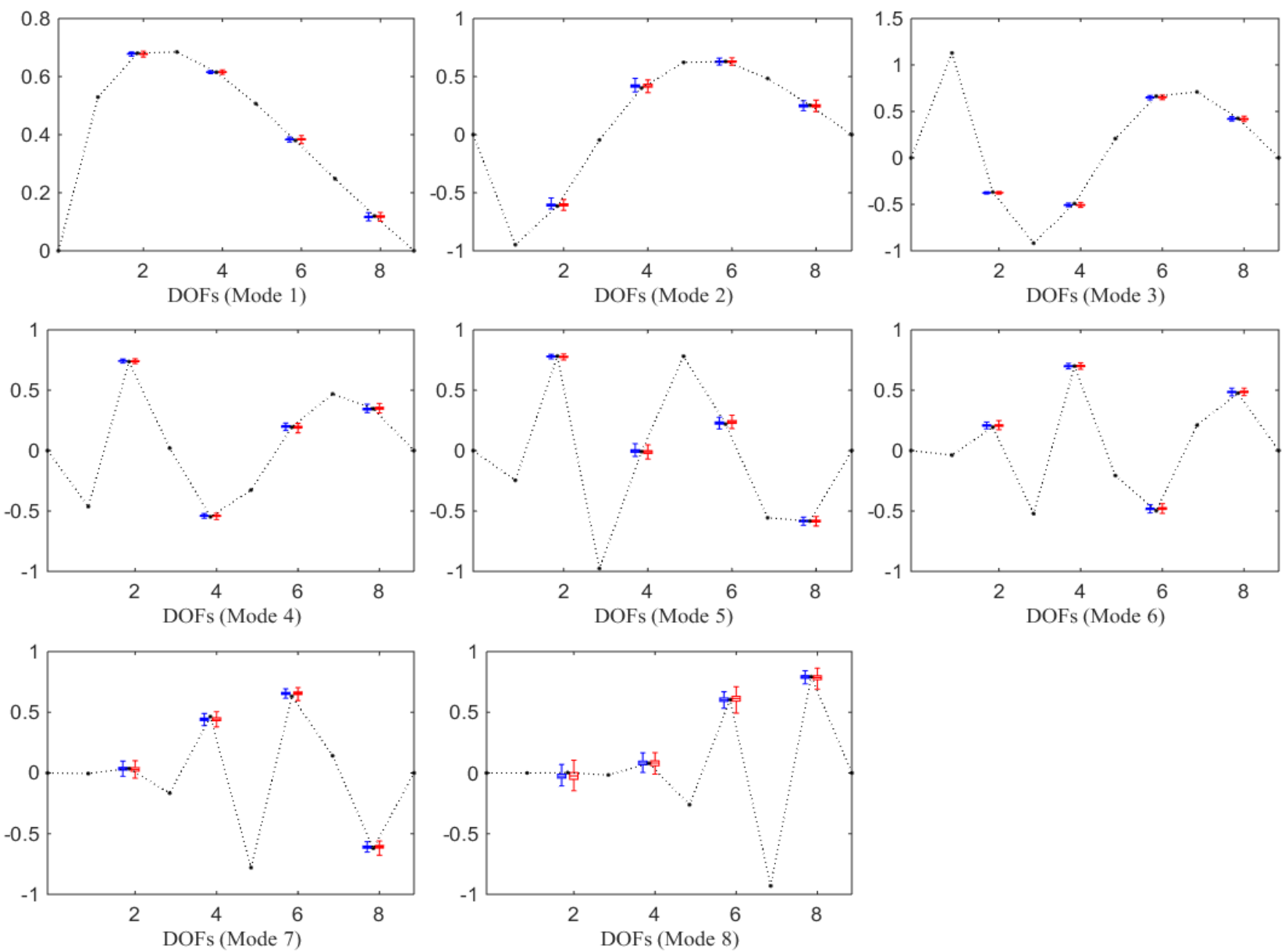

Fig. 9 Boxplot of identified mode shapes

Blue: variational Bayes; Red: Gibbs sampler; Dot line: true value 

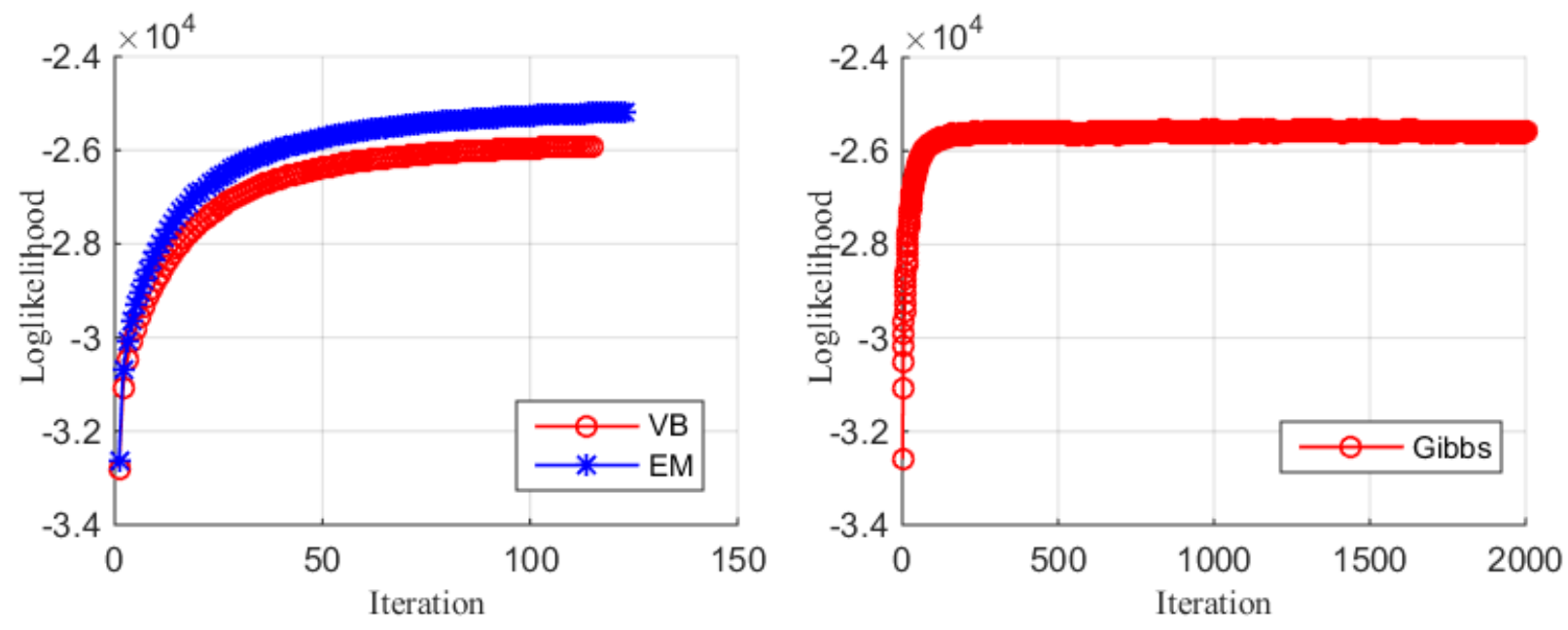

Fig. 10 Convergence of VB, EM and Gibbs sampler

We have shown that the VB estimation is consistent and can provide an approximated posterior distribution; however, as an iterative method, it is computationally demanding, especially when many iterations are needed to converge. Wang \& Titterington [25] have proved almost linear convergence of the VB algorithm for the exponential family of distributions. Here, we empirically investigate its convergence property, as shown in Fig. 10. The VB converges a little faster than the EM algorithm. Since the EM algorithm has a linear convergence rate [33], this shows that the VB converges at least with a linear rate. For comparison, the convergence curve of the Gibbs sampler is also shown in Fig. 10. It is observed that the Gibbs sampler converges at about 200 iterations, but much more iterations are needed to provide enough samples to estimate the distribution. In general, the Gibbs sampler is more computationally demanding.

In state-space model-based modal identification, one has to specify the order of the system, i.e. two times of the number of physical modes. Since we do not know the exact order in advance, one favored practice is to specify a high order and then to identify the physical modes from the estimated model. In this paper, we do not solve this problem rigorously, but only provide an empirical evidence that the physical modes can be identified based on the uncertainty information. For this 8-DOF mass-spring system, an order of 24 is specified in the state-space model, so that there are 4 spurious modes. Figure 11 shows the COVs of identified frequencies and standard deviation of damping ratios for both the VB and Gibbs sampler. Spurious modes correspond to the peaks in the graph, i.e. spurious modes have significantly larger uncertainties than the physical modes. The intuitive explanation for this phenomenon, similar to that of a stabilization diagram [5], is that the physical modes should be consistent in different realizations, while the spurious computational and noise modes have no reason to be focused. 

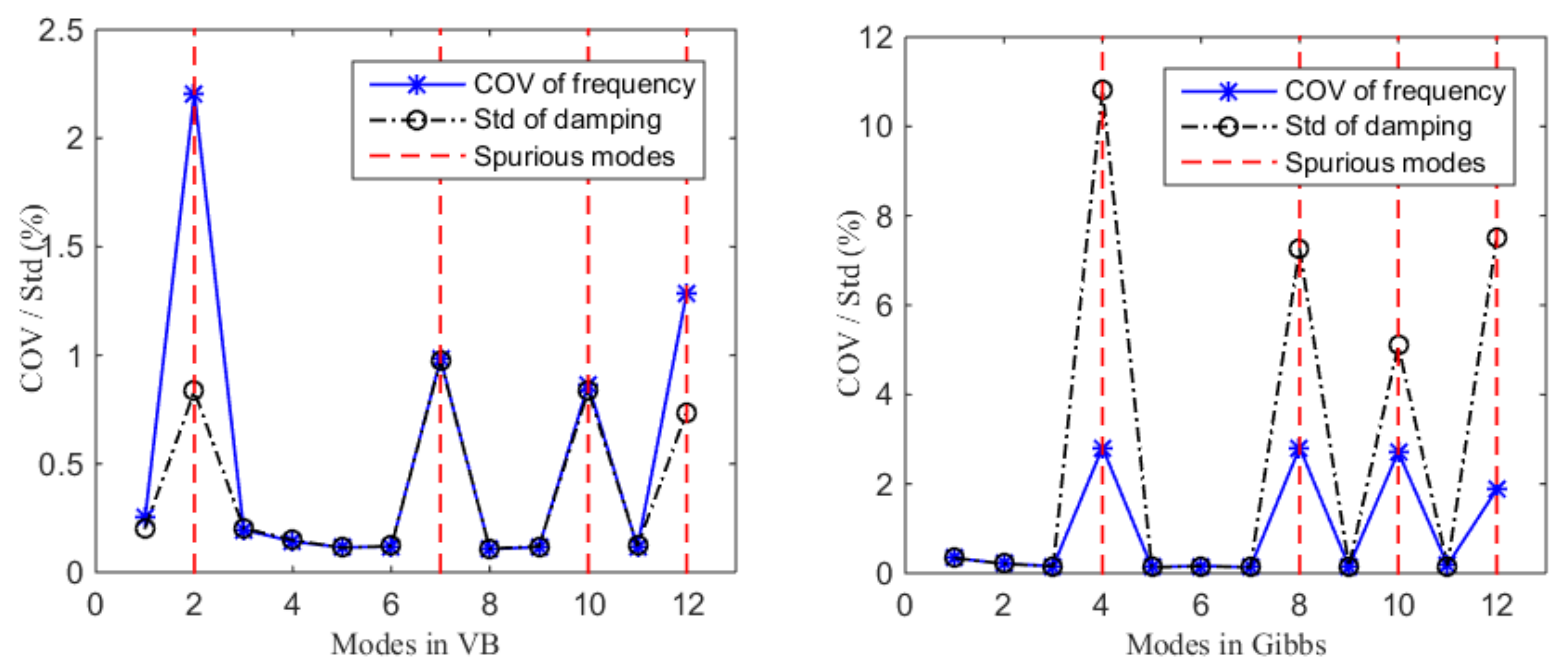

Fig. 11 Spurious modes identification based on uncertainty

\subsection{One Rincon Hill Tower}

The One Rincon Hill Tower (ORHT), a landmark building in San Francisco, California, is a 64-story reinforced-concrete, shear-wall building. It is also the tallest building in the United States that is designed using performance-based seismic design procedures. In order to evaluate the design and assess the condition of the building after a future earthquake, extensive seismic instrumentation of the building was jointly conducted by the California Strong Motion Instrumentation Program and the National Strong Motion Project and managed by United States Geological Survey [36]. A 72-channel seismic monitoring system (Fig. 12) was installed to stream real-time acceleration data throughout the building. Two sets of recorded ambient vibration time series are available in the website of the Center for Engineering Strong Motion Data (www.strongmotioncenter.org).

There are a total of 72 accelerometers, of which 4 measure vertical motions and are not considered in this study, since only the lateral modes are of interest. Furthermore, in order to capture the 3D motion of the building, only levels with 3 accelerometers, one in north-south (NS) and two in east-west (EW1 and EW2) directions of each floor, are included in the analysis. The raw data has a sampling frequency of $200 \mathrm{~Hz}$ and a duration of 230 seconds. According to Ref. [36], modes with frequencies below $7 \mathrm{~Hz}$ are of interest. Therefore, for the sake of reducing the computational burden, the raw data is resampled down to $20 \mathrm{~Hz}$ so that all higher modes are filtered out.

Initialized with the parameters identified by the SSI algorithm [7] with a model order of 120, the EM, VB and Gibbs sampler are used for the OMA of the ORHW. Figure 13 shows the iteration steps of each algorithm with a convergence criterion equal to $\rho=10^{-4}$, where 178 iterations (about 115 min on a Digital Storm laptop with Intel ${ }^{\circledR}$ Core $^{\mathrm{TM}}$ i7 CPU @2.50 GHz and RAM 16.0 GB) are spent for the EM, 84 iterations (about $55 \mathrm{~min}$ ) are spent for VB, and 800 samples (about $534 \mathrm{~min}$ ) are used for the Gibbs sampler. One may argue that 800 samples are not enough for the Gibbs sampler, but running more than 10 hours was not practical. One must admit that, while the VB is the most efficient algorithm, all three are perhaps too computationally demanding for practical use. One way to accelerate the computation is to loosen the convergence criterion. 

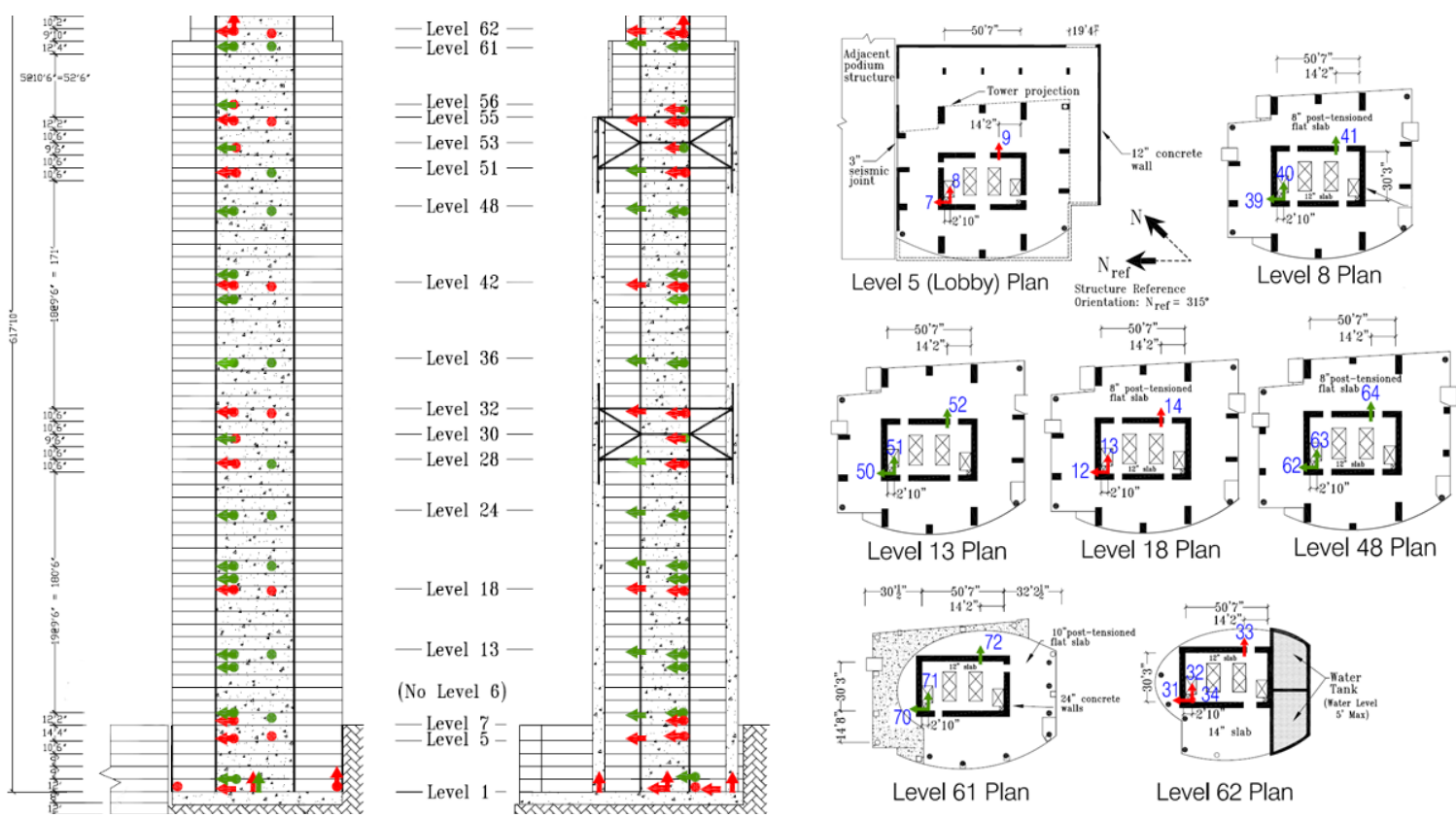

Fig. 12 Configuration of accelerometers along the vertical and plan of the building (www.strongmotioncenter.org).
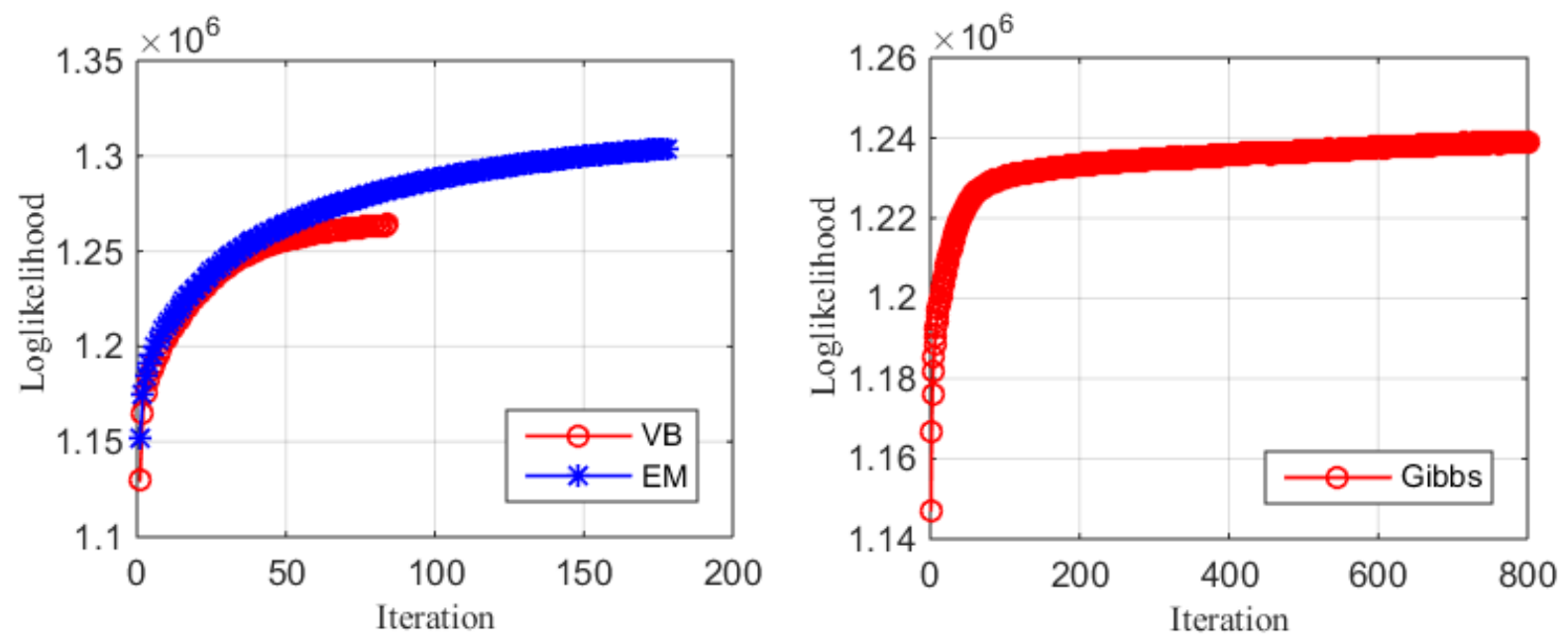

Fig. 13 Convergence of EM, VB and Gibbs sampler in OMA of ORHT

Identification of spurious modes is shown in Fig. 14 for VB and Gibbs sampler, where the averaged power spectral density of all locations is also plotted. When the order of the model is high, plots of the COV of frequencies and the standard deviation of damping ratios might be too complex to allow accurate identification of the spurious mode. To address this problem, more requirements should be enforced. Here, we require parameter $\lambda_{\min }$ to be less than 0.01 , i.e., that the imaginary part of the mode shape not be too large. The second requirement is that each mode corresponds to a peak in the average power spectral density (PSD) over all measurements. 

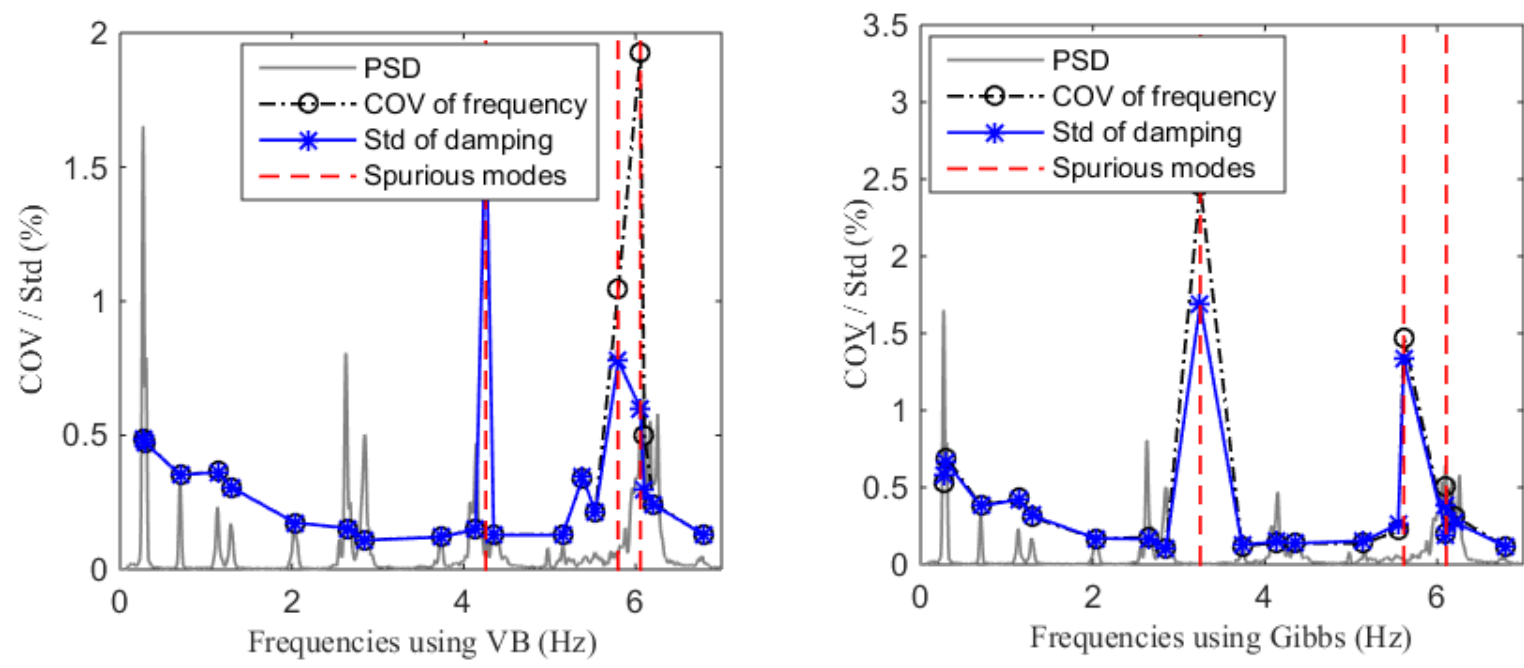

Fig. 14 Identification of spurious modes in VB and Gibbs sampler PSD: scaled power spectrum density

Tab.2 Identified Modal Parameters using EM, VB and Gibbs Sampler

\begin{tabular}{|c|c|c|c|c|c|c|c|c|c|c|c|c|c|}
\hline \multirow{3}{*}{ Mode } & \multirow{2}{*}{\multicolumn{5}{|c|}{ Frequencies $(\mathrm{Hz})$}} & \multicolumn{7}{|c|}{ Damping Ratios (\%) } & \multirow{3}{*}{ Mode shape } \\
\hline & \multirow{2}{*}{ EM } & & & & & \multirow{2}{*}{ EM } & \multicolumn{3}{|c|}{ VB } & \multicolumn{3}{|c|}{ Gibbs } & \\
\hline & & Mean & $\operatorname{COV}(\%)$ & Mean & $\operatorname{COV}(\%)$ & & Mean & STD & $\operatorname{COV}(\%)$ & Mean & STD & $\operatorname{COV}(\%)$ & \\
\hline 1 & 0.27 & 0.27 & 0.49 & 0.27 & 0.58 & 1.01 & 0.96 & 0.49 & 51.04 & 1.09 & 0.53 & 48.62 & 1st EW \\
\hline 2 & 0.30 & 0.30 & 0.48 & 0.30 & 0.67 & 1.60 & 0.94 & 0.48 & 51.06 & 1.86 & 0.67 & 36.02 & 1 st NS \\
\hline 3 & 0.70 & 0.70 & 0.35 & 0.70 & 0.38 & 1.39 & 1.30 & 0.36 & 27.69 & 1.22 & 0.38 & 31.15 & 1st Torsion \\
\hline 4 & 1.14 & 1.14 & 0.37 & 1.14 & 0.42 & 2.97 & 2.10 & 0.36 & 17.14 & 2.71 & 0.43 & 15.87 & 2nd EW \\
\hline 5 & 1.30 & 1.30 & 0.30 & 1.30 & 0.32 & 2.02 & 1.75 & 0.30 & 17.14 & 1.71 & 0.31 & 18.13 & 2nd NS \\
\hline 6 & 2.03 & 2.03 & 0.17 & 2.03 & 0.17 & 1.03 & 0.90 & 0.17 & 18.89 & 0.76 & 0.17 & 22.37 & 2nd Torsion \\
\hline 7 & 2.64 & 2.65 & 0.15 & 2.65 & 0.17 & 1.11 & 0.92 & 0.15 & 16.3 & 1.02 & 0.18 & 17.65 & 3rd EW \\
\hline 8 & 2.85 & 2.85 & 0.11 & 2.85 & 0.11 & 0.59 & 0.51 & 0.11 & 21.57 & 0.45 & 0.11 & 24.44 & 3rd NS \\
\hline 9 & 3.74 & 3.73 & 0.12 & 3.73 & 0.13 & 0.96 & 0.82 & 0.12 & 14.63 & 0.80 & 0.12 & 15.00 & 3rd Torsion \\
\hline 10 & 4.14 & 4.14 & 0.15 & 4.13 & 0.15 & 1.13 & 1.23 & 0.15 & 12.20 & 1.14 & 0.14 & 12.28 & 4th EW \\
\hline 11 & 4.35 & 4.35 & 0.13 & 4.34 & 0.14 & 0.90 & 1.00 & 0.13 & 13.00 & 1.03 & 0.14 & 13.59 & 4th NS \\
\hline 12 & 5.15 & 5.15 & 0.13 & 5.15 & 0.15 & 1.11 & 1.08 & 0.13 & 12.04 & 1.12 & 0.14 & 12.50 & 4th Torsion \\
\hline 13 & 5.31 & 5.39 & 0.33 & - & - & 3.95 & 4.64 & 0.33 & 7.11 & - & - & - & 5th EW \\
\hline 14 & 5.54 & 5.53 & 0.22 & 5.54 & 0.29 & 2.14 & 2.17 & 0.21 & 9.68 & 2.21 & 0.23 & 10.41 & 5th NS \\
\hline 15 & 6.11 & 6.10 & 0.27 & 6.10 & 0.20 & 1.66 & 1.66 & 0.27 & 16.27 & 1.26 & 0.20 & 15.87 & 6th EW \\
\hline 16 & 6.20 & 6.21 & 0.25 & 6.21 & 0.28 & 1.76 & 1.63 & 0.26 & 15.95 & 1.70 & 0.32 & 18.82 & 6th NS \\
\hline 17 & 6.81 & 6.80 & 0.13 & 6.78 & 0.13 & 1.15 & 1.08 & 0.13 & 12.04 & 0.97 & 0.12 & 12.37 & 5th Torsion \\
\hline
\end{tabular}

After removal of spurious modes, the identified physical modes are listed in Table 2. The three approaches yield similar modal parameters, indicating the consistency of the VB approach. It is noted that Çelebi et al. [36] only identified 12 modes using the SSI algorithm; 5 additional modes are identified in this study. As for the uncertainty estimation, compared to the previous example, the COVs of VB are closer to those of the Gibbs sampler, indicating that the VB may perform better for real data. Figures 15 and 16 show the CDFs and empirical CDFs of modal frequencies and damping ratios, respectively. Generally speaking, the CDFs identified by VB are quite close to those by the Gibbs sampler, and some of them are almost identical. The mode shapes are illustrated in Fig. 17, though the uncertainty information is not shown for the sake of 
succinctness. Again, we see that the COVs of the estimated frequencies are approximately equal to the standard deviations of the estimated damping ratios.
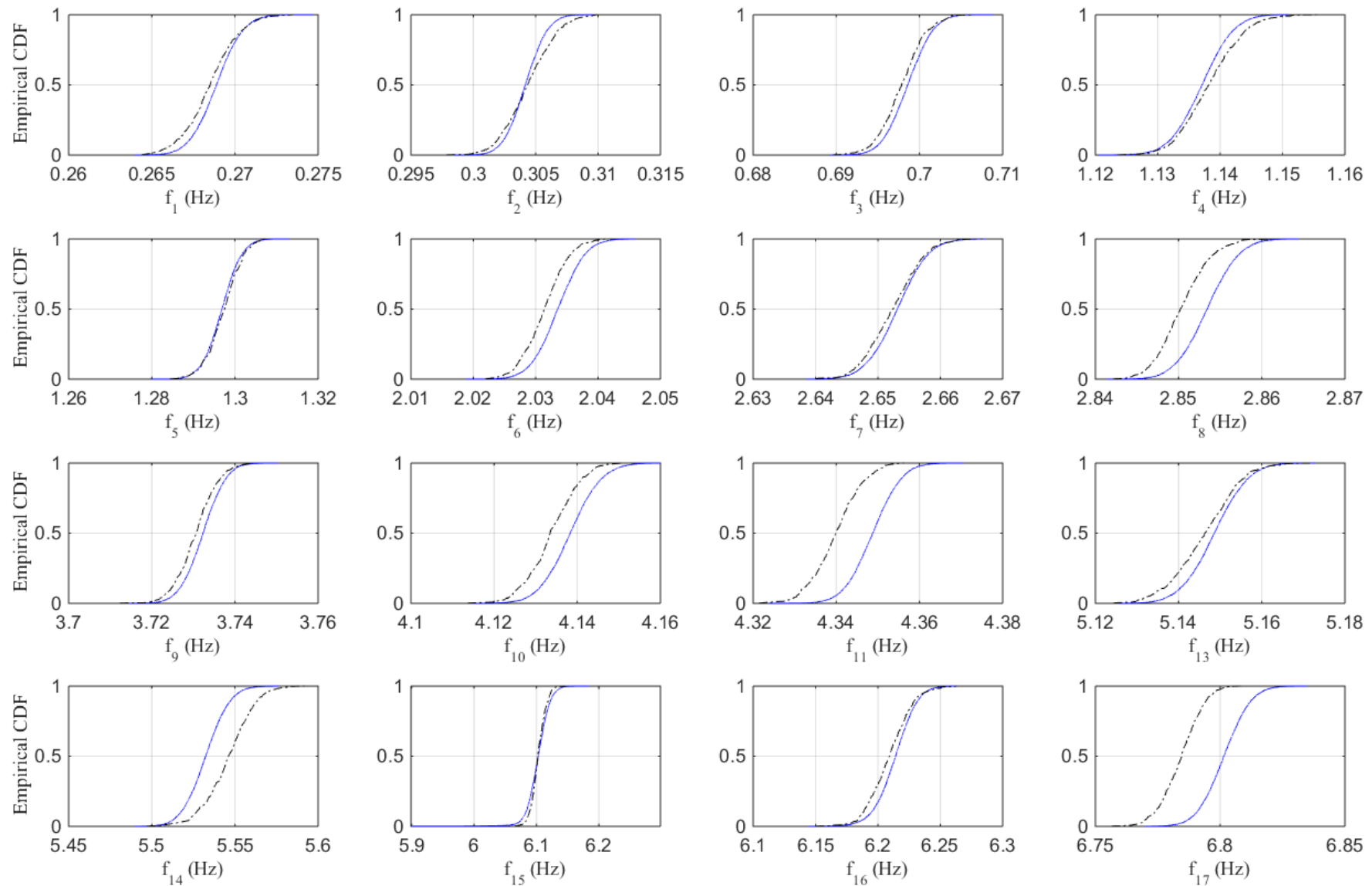

Fig. 15 Empirical CDF of identified modal frequencies Solid line: VB; Dash-dot line: Gibbs sampler 

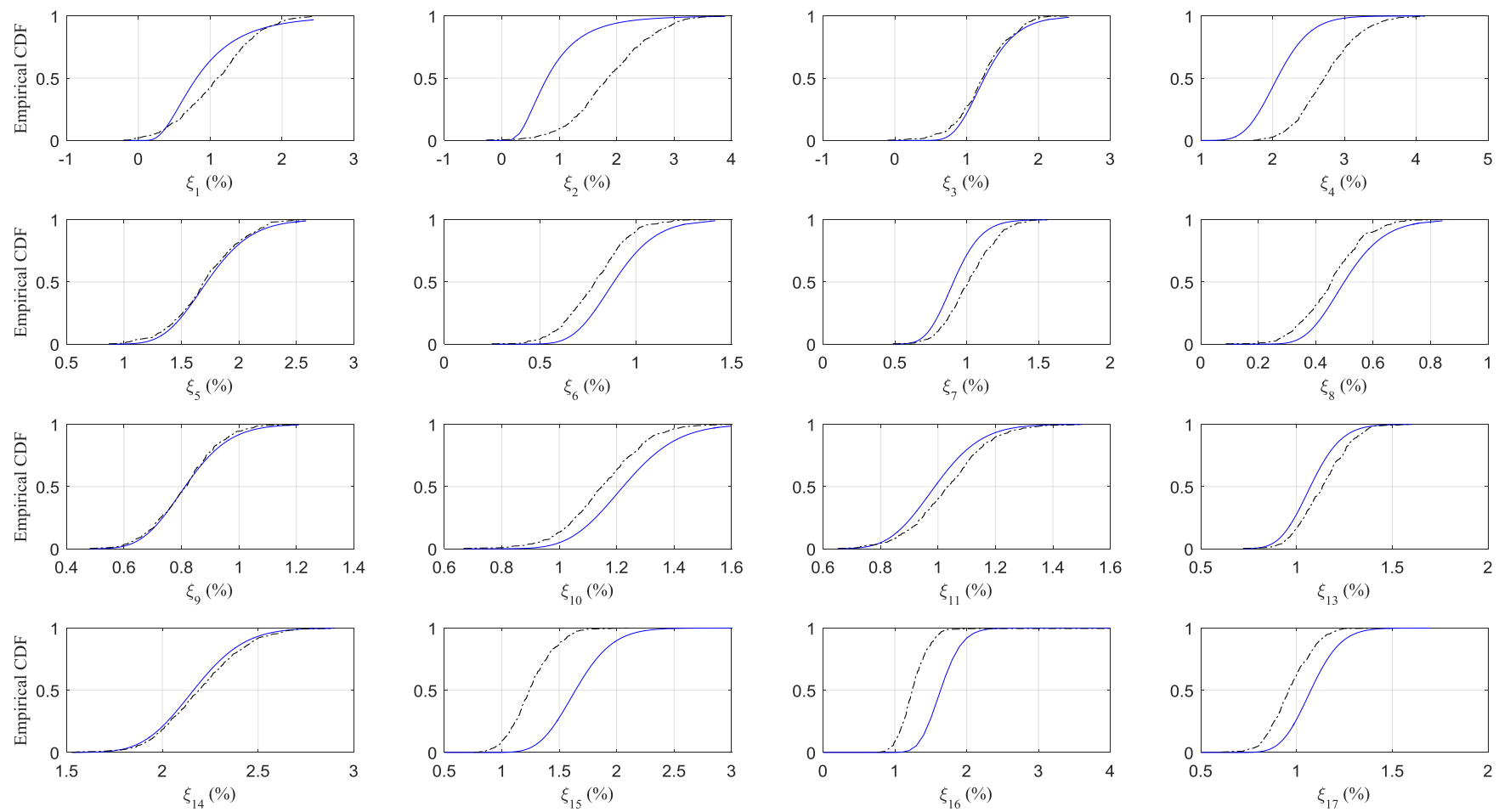

Fig. 16 Empirical CDF of identified damping ratios Solid line: VB; Dash-dot line: Gibbs sampler 

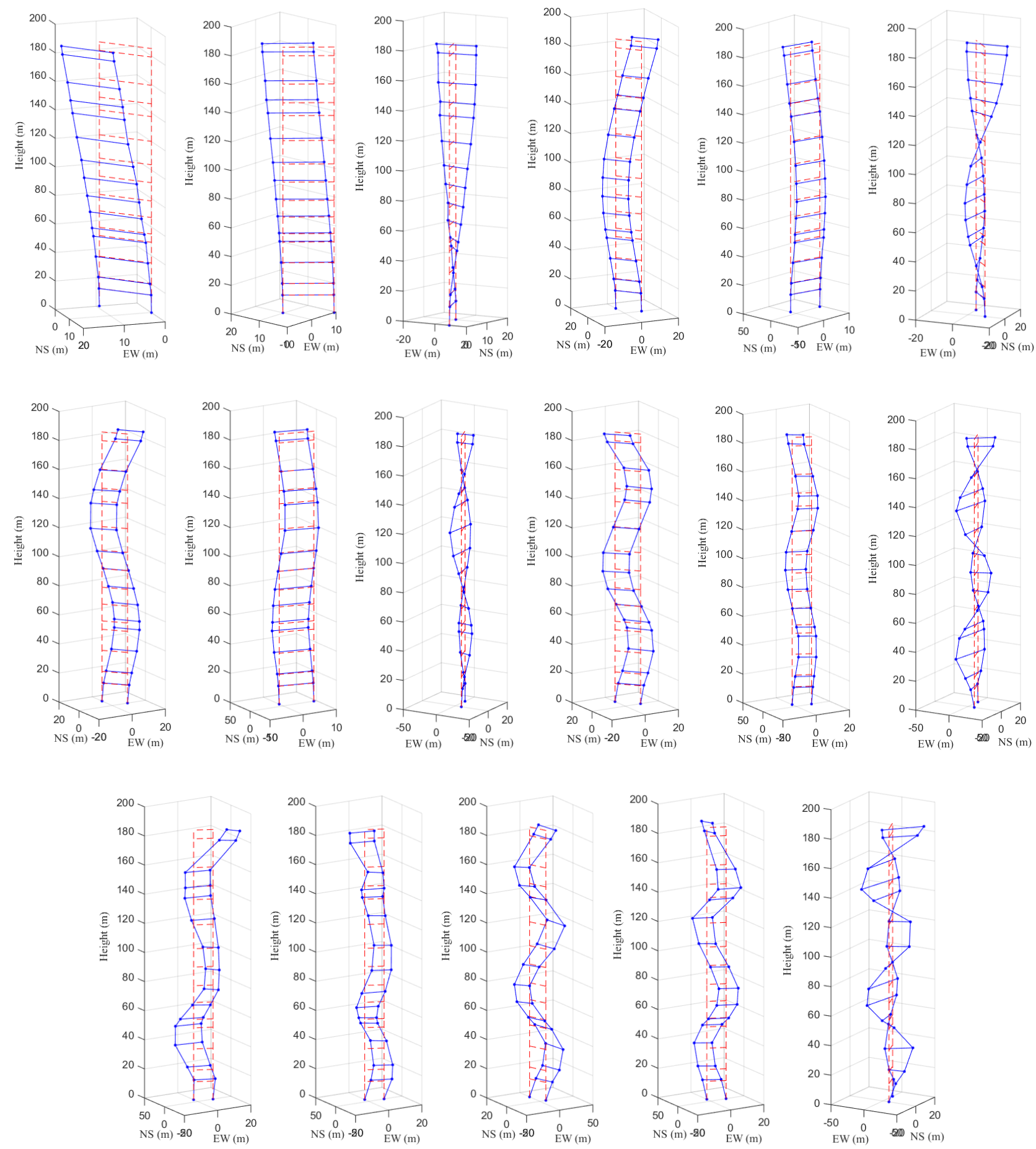

Fig. 17 Mode shapes identified using VB.

\section{Conclusions}

An operational modal analysis approach based on variational Bayes is introduced. Starting from the statespace representation of the structural system and making the mean-field approximation that neglects the 
statistical dependence between the model parameters and the latent variables, the joint posterior distribution of the state-transition matrix and observation matrix is formulated, while accounting for the effects of process noise and measurement error. Since there is no closed-form solution for the distributions of the eigenvalues and eigenvectors of a random matrix, the first-order Taylor series expansion is applied to give an approximated posterior distribution of modal parameters. Through the empirical study of the OMA of an 8DOF mass-spring system and the One Rincon Hill Tower (ORHT), the following conclusions are derived:

(1) The VB approach works in the sense of coordinate ascent and neither gradient nor Hessian matrix are required in the optimization scheme. The computational burden is less than that of EM and Gibbs sampler, but still heavy for real projects if there are many sensors and a large number of structural modes.

(2) The posterior mean obtained by VB is consistent with results obtained by Expectation-Maximization and by Gibbs sampler. Compared to the Gibbs sampler, the VB tends to underestimate the variability in the estimated modal parameters, but the difference is small for the ORHT.

(3) The approximated posterior distribution of modal parameters coincides well with samples generated from the posterior distribution of the state-transition and observation matrices, especially for the frequencies and damping ratios. It is found that the coefficient of variation of the frequency is approximately equal to the standard deviation of the damping ratio in the same mode.

(4) The removal of spurious modes is empirically studied based upon the uncertainty information and promising results are shown. A more rigorous treatment of the removal of spurious modes requires further study.

(5) Of the three approaches studied in this paper, the VB algorithm is recommended. The EM algorithm only provides a point estimate and its convergence is slower. The computational demand of the Gibbs sampler is too high for application in practice.

\section{Acknowledgements}

Support from the National Science Foundation under Grant No. CMMI-1130061 is gratefully acknowledged.

\section{References}

[1] W. Yan, L.S. Katafygiotis, A novel Bayesian approach for structural model updating utilizing statistical modal information from multiple setups, Structural Safety, 52(B) (2015) 260-271.

[2] R. Kumar, T. Singh, Design of input shapers using modal cost for multi-mode systems, Automatica, 46 (3) (2010) $598-604$.

[3] M. Döhler, L. Mevel, Subspace-based fault detection robust to changes in the noise covariances, Automatica, 49 (9) (2013) 2734-2743.

[4] B.J.A Costa, F. Magalhães, Á. Cunha, J. Figueiras, Rehabilitation assessment of a centenary steel bridge based on modal analysis, Engineering Structures, 56 (2013) 260-272.

[5] E. Reynders, System identification methods for (operational) modal analysis: review and comparison. Archives of Computational Methods in Engineering, 19 (1) (2012) 51-124.

[6] G.H. James, T.G. Carne, J.P. Lauffer, A.R. Nord, Modal testing using natural excitation, in: Proceedings of 10th International Modal Analysis Conference, San Diego, USA, 1992.

[7] P. Van Overschee, B. De Moor, Subspace algorithms for the stochastic identification problem, Automatica, 29 (3) (1993) 649660.

[8] L. Ljung, System Identification: Theory for the User, 2nd ed., Prentice Hall PTR, Upper Saddle River, USA, 1999. 
[9] R. Brincker, L. Zhang, P. Andersen, Modal identification from ambient response using frequency domain decomposition, in: Proceedings of IMAC18, San Antonio, USA, 2000.

[10] P. Guillaume, P. Verboven, S. Vanlanduit, H. Van der Auweraer, B. Peeters, A Poly-reference implementation of the leastsquares complex frequency domain estimator, in: Proceedings of IMAC21, Kissimmee, USA, 2003.

[11] K. Ciloglu, Y. Zhou, F, Moon, A.E. Aktan, Impacts of epistemic uncertainty in operational modal analysis, Journal of Engineering Mechanics, 138 (9) (2012) 1059-1070.

[12] X.-B. Lam, L. Mevel, Uncertainty quantification for eigensystem-realization-algorithm, a class of subspace system identification, in: Proceeding of the 18th IFAC World Congress, Milan, Italy, 2011.

[13] E. Reynders, R. Pintelon, G. De Roeck, Uncertainty bounds on modal parameters obtained from stochastic subspace identification, Mechanical Systems and Signal Processing, 22 (2008) 948 - 969.

[14] M. Dohler, X.-B. Lam, L. Mevel, Uncertainty quantification for stochastic subspace identification on multi-setup measurements, in: Proceeding of 50th IEEE Conference on CDC-ECC, Orlando, USA, 2011 pp. 6451-6456.

[15] V.H. Vu, M. Thomas, Uncertainties on modal parameters by operational modal analysis, Mechanics \& Industry, 15 (2014) 153-158.

[16] M.J. Wainwright, M.I. Jordan, Graphical models, exponential families, and variational Inference, Foundations and Trends in Machine Learning, 1 (2008) 1-305.

[17] F.J. Cara, J. Carpio, J. Juan, E. Alarcón, An approach to operational modal analysis using the expectation maximization algorithm, Mechanical Systems and Signal Processing, 31 (2012) 109-129.

[18] M. El-kafafy, T. De Troyer, B. Peeters, P. Guillaume, Fast maximum-likelihood identification of modal parameters with uncertainty intervals: a modal model-based formulation, Mechanical Systems and Signal Processing, 37 (2013) 422-439.

[19] K.-V. Yuen, L.S. Katafygiotis, Bayesian time-domain approach for modal updating using ambient data, Probabilistic Engineering Mechanics, 16 (2001) 219-231.

[20] S.-K. Au, Uncertainty law in ambient modal identification-Part I: Theory, Mechanical Systems and Signal Processing, 48 (2014) 15-33.

[21] F. Lindsten, T.B. Schön, M.I. Jordan, Bayesian semiparametric Wiener system identification, Automatica, 49 (7) (2013), 2053-2063.

[22] M.J. Beal, Variational algorithms for approximate bayesian inference, Ph.D. Dissertation, Gatsby Computational Neuroscience Unit, University College London, 2003.

[23] K. Fujimoto, A. Satoh, S. Fukunaga, System identification based on variational Bayes method and the invariance under coordinate transformations, in: Proceeding of 50th IEEE Conference on CDC-ECC, Orlando, USA, 2011, pp. 3882-3888.

[24] A.K. Gupta, D. Nagar, Matrix Variate Distributions, Chapman \& Hall/CRC, Boca Raton, USA, 1999.

[25] B. Wang, D.M. Titterington, Convergence and asymptotic normality of variational Bayesian approximations for exponential family models with missing values, in: Proceedings of the 20th conference on Uncertainty in artificial intelligence, Banff, Canada, 2004, pp. 577-584.

[26] R.W. Keener, Theoretical Statistics: Topics for a Core Course, Springer, New York, USA, 2010.

[27] J. Grimmer, An introduction to Bayesian Inference via variational approximations, Political Analysis, 19 (1) (2010) 32-47.

[28] G.F. Franklin, J.D. Powell, M.L. Workman, Digital Control of Dynamic Systems, 3rd ed., Ellis-Kagle Press, Half Moon Bay, USA, 1997.

[29] F.M. Callier, C.A. Desoer, Linear System Theory, Springer Science+Business Media, LLC, New York, USA, 1991.

[30] D. Barber, S. Chiappa, Unified inference for variational Bayesian linear Gaussian state-space model, in: Proceedings of NIPS 19, Vancouver, Canada, 2006 pp. 81-88.

[31] R.H. Shumway, D.S. Stoffer, Time Series Analysis and Its Applications: With R Examples, 3rd ed., Springer, New York, USA, 2011.

[32] G. McLachlan, K. Thriyambakam,. The EM algorithm and extensions. John Wiley \& Sons, 2007.

[33] S. Gibson, B. Ninness, Robust maximum-likelihood estimation of multivariable dynamic systems, Automatica, 4 (2005) $1667-1682$.

[34] S. Adhikari, M.I. Friswell, Random matrix eigenvalue problems in structural dynamics, International Journal for Numerical Methods in Engineering, 69 (3) (2007) 562-591.

[35] F.J. Cara, J. Juan, E. Alarcón, E. Reynders, G. De Roeck, Modal contribution and state space order selection. Mechanical Systems and Signal Processing, 38 (2013) 276-298.

[36] M. Çelebi, M. Huang, A. Shakal, J. Hooper, R. Klemencic, Ambient response of a unique performance-based design tall building with dynamic response modification features. Structural Design of Tall and Special Buildings, 22 (2013) 816-829.

[37] J. Vlach, K. Singhal, Computer Methods for Circuit Analysis and Design, Springer Science \& Business Media, New York,

USA, 1994. 


\section{Appendix A: Technical lemmas}

\section{Definition A.1 Matrix normal distribution}

The random matrix $\boldsymbol{\Gamma} \in \mathbb{R}^{p \times n}$ is said to have a matrix normal distribution $\operatorname{MN}\left(\boldsymbol{\mu}_{\boldsymbol{\Gamma}}, \boldsymbol{\Sigma}, \boldsymbol{\Pi}\right)$, with mean matrix $\boldsymbol{\mu}_{\boldsymbol{\Gamma}} \in \mathbb{R}^{p \times n}$ and left and right covariance matrices $\boldsymbol{\Sigma} \in \mathbb{R}^{p \times p}$ and $\boldsymbol{\Pi} \in \mathbb{R}^{n \times n}$, respectively, when $\operatorname{vec}(\boldsymbol{\Gamma}) \sim \operatorname{MVN}\left(\operatorname{vec}\left(\boldsymbol{\mu}_{\boldsymbol{\Gamma}}\right), \boldsymbol{\Pi} \otimes \boldsymbol{\Sigma}\right)$, where $\operatorname{vec}(\cdot)$ means stacking the columns of the matrix into a vector, MVN denotes the multivariate normal distribution, and ' $\Theta$ ' is the Kronecker product. The $\operatorname{PDF}$ of $\operatorname{MN}\left(\boldsymbol{\mu}_{\Gamma}, \boldsymbol{\Sigma}, \boldsymbol{\Pi}\right)$ is given by

$$
p_{\Gamma}(\boldsymbol{\gamma})=\frac{\exp \left\{-\frac{1}{2} \operatorname{tr}\left[\boldsymbol{\Pi}^{-1}\left(\boldsymbol{\gamma}-\boldsymbol{\mu}_{\Gamma}\right)^{\mathrm{T}} \boldsymbol{\Sigma}^{-1}\left(\boldsymbol{\gamma}-\boldsymbol{\mu}_{\boldsymbol{\Gamma}}\right)\right]\right\}}{(2 \pi)^{n p / 2} \operatorname{det}(\boldsymbol{\Sigma})^{n / 2} \operatorname{det}(\boldsymbol{\Pi})^{p / 2}}
$$

\section{Definition A.2 Inverse Wishart distribution}

The random matrix $\boldsymbol{\Sigma} \in \mathbb{R}^{p \times p}$ is said to be distributed as inverse Wishart, $\operatorname{IW}(d, \boldsymbol{D})$, with $d(>p-1)$ degrees of freedom and positive definite scale matrix $\boldsymbol{D}$ when its PDF is given by

$$
p_{\Sigma}(\boldsymbol{\sigma})=\frac{\operatorname{det}(\boldsymbol{D})^{d / 2} \operatorname{det}(\boldsymbol{\sigma})^{-(d+p+1) / 2}}{2^{d p / 2} \Gamma_{p}(d / 2)} \exp \left[-\frac{1}{2} \operatorname{tr}\left(\boldsymbol{D} \boldsymbol{\sigma}^{-1}\right)\right]
$$

in which $\Gamma_{p}(\cdot)$ is the multivariate gamma function.

\section{Definition A.3 Matrix variate- $t$ distribution}

The random matrix $\boldsymbol{T} \in \mathbb{R}^{p \times n}$ is said to have a matrix variate- $t$ distribution, $\operatorname{MVT}\left(m, \boldsymbol{\mu}_{\boldsymbol{T}}, \boldsymbol{\Sigma}, \boldsymbol{\Pi}\right)$, with mean matrix $\boldsymbol{\mu}_{\boldsymbol{T}} \in \mathbb{R}^{p \times n}$, left and right covariance matrices $\boldsymbol{\Sigma} \in \mathbb{R}^{p \times p}$ and $\boldsymbol{\Pi} \in \mathbb{R}^{n \times n}$, respectively, and $m(>0)$ degrees of freedom when its PDF is given by

$$
p_{\boldsymbol{T}}(\boldsymbol{t})=\frac{\Gamma_{p}((m+n+p-1) / 2)}{\pi \Gamma_{p}((m+p-1) / 2)} \operatorname{det}(\boldsymbol{\Sigma})^{-\frac{n}{2}} \operatorname{det}(\boldsymbol{\Pi})^{-\frac{p}{2}} \operatorname{det}\left[\boldsymbol{I}_{p}+\boldsymbol{\Sigma}^{-1}\left(\boldsymbol{t}-\boldsymbol{\mu}_{\boldsymbol{T}}\right) \boldsymbol{\Pi}^{-1}\left(\boldsymbol{t}-\boldsymbol{\mu}_{\boldsymbol{T}}\right)^{\mathrm{T}}\right]^{-\frac{m+n+p-1}{2}}
$$

Lemma A.1 Let random matrix $\boldsymbol{\Sigma} \in \mathbb{R}^{p \times p}$ have the inverse Wishart $\operatorname{IW}(d, \boldsymbol{D})$ distribution. Given $\boldsymbol{\Sigma}$, if the conditional distribution of random matrix $\boldsymbol{\Gamma} \in \mathbb{R}^{p \times n}$ follows $\operatorname{MN}\left(\boldsymbol{\mu}_{\boldsymbol{\Gamma}}, \boldsymbol{\Sigma}, \boldsymbol{\Pi}\right)$, then the marginal distribution of $\boldsymbol{\Gamma}$ follows a matrix variate- $t$ distribution $\operatorname{MVT}\left(d-p+1, \boldsymbol{\mu}_{\boldsymbol{\Gamma}}, \boldsymbol{D}, \boldsymbol{\Pi}\right)$.

Lemma A.2 Let random matrix $\boldsymbol{T} \in \mathbb{R}^{p \times n}$ have the matrix variate- $t$ distribution $\operatorname{MVT}\left(m, \boldsymbol{\mu}_{\boldsymbol{T}}, m \boldsymbol{\Sigma}, \boldsymbol{\Pi}\right)$, then $\stackrel{\mathcal{D}}{\rightarrow} \boldsymbol{\Gamma}$ as $n \rightarrow \infty$ where random matrix $\boldsymbol{\Gamma} \in \mathbb{R}^{p \times n}$ follows a matrix normal distribution $\operatorname{MN}\left(\boldsymbol{\mu}_{\boldsymbol{\Gamma}}, \boldsymbol{\Sigma}, \boldsymbol{\Pi}\right)$ and $\stackrel{\mathcal{D}}{\rightarrow}$ denotes convergence in distribution.

A proof of the above lemmas can be found in Ref. [23]. 


\section{Appendix B: Proofs of Theorem 2 and Lemma 5.1}

Proof of Theorem 2.

(1) Replacing $\boldsymbol{X}, \boldsymbol{Y}$ and $\boldsymbol{\Theta}$ in Eqn. (4) by $\boldsymbol{X}_{1: N+1}, \boldsymbol{Y}_{1: N}$ and $\{\boldsymbol{\Gamma}, \boldsymbol{\Sigma}\}$, we have

$$
\begin{aligned}
& \mathrm{F}\left[q_{\boldsymbol{\Theta}}(\boldsymbol{\theta}), q_{X_{1: N+1}}\left(\boldsymbol{x}_{1: N+1}\right)\right]=-\iint q_{\Gamma \Sigma}(\boldsymbol{\gamma}, \boldsymbol{\sigma}) \ln \frac{q_{\Gamma \Sigma}(\gamma, \boldsymbol{\sigma})}{\pi_{\Gamma \Sigma}(\gamma, \boldsymbol{\sigma})} d \boldsymbol{\gamma} d \boldsymbol{\sigma}-\int q_{\boldsymbol{X}_{1: N+1}}\left(\boldsymbol{x}_{1: N+1}\right) \ln \frac{q_{X_{1: N+1}}\left(\boldsymbol{x}_{1: N+1}\right)}{\pi_{X_{1: N+1}}\left(\boldsymbol{x}_{1 N+1}\right)} d \boldsymbol{x}_{1: N+1} \\
& +\iiint q_{\Gamma \Sigma}(\boldsymbol{\gamma}, \boldsymbol{\sigma}) q_{\boldsymbol{X}_{1: N+1}}\left(\boldsymbol{x}_{1: N+1}\right) \ln p_{\boldsymbol{Y}_{1: N} \mid \boldsymbol{\Gamma} \Sigma \boldsymbol{X}_{1: N+1}}\left(\boldsymbol{y}_{1: N} \mid \boldsymbol{\gamma}, \boldsymbol{\sigma}, \boldsymbol{x}_{1: N+1}\right) d \boldsymbol{\gamma} d \boldsymbol{\sigma} d \boldsymbol{x}_{1: N+1} \\
& =-\iint q_{\Gamma \Sigma}(\boldsymbol{\gamma}, \boldsymbol{\sigma}) \ln \frac{q_{\Gamma \Sigma}(\gamma, \boldsymbol{\sigma})}{p_{\Gamma \Sigma}(\gamma, \boldsymbol{\sigma})} d \boldsymbol{\gamma} d \boldsymbol{\sigma}-\int q_{X_{1: N+1}}\left(\boldsymbol{x}_{1: N+1}\right) \ln \frac{q_{X_{1: N+1}}\left(\boldsymbol{x}_{1: N+1}\right)}{\pi_{X_{1}}\left(\boldsymbol{x}_{1}\right)} d \boldsymbol{x}_{1: N+1} \\
& +\iiint q_{\Gamma \Sigma}(\boldsymbol{\gamma}, \boldsymbol{\sigma}) q_{\boldsymbol{X}_{1: N+1}}\left(\boldsymbol{x}_{1: N+1}\right) \sum_{k=1}^{N} \ln p_{\boldsymbol{Z}_{k} \mid \boldsymbol{\Gamma} \boldsymbol{\Sigma} \boldsymbol{X}_{k}}\left(\mathbf{z}_{k} \mid \boldsymbol{\gamma}, \boldsymbol{\sigma}, \boldsymbol{x}_{k}\right) d \boldsymbol{\gamma} d \boldsymbol{\sigma} d \boldsymbol{x}_{k}
\end{aligned}
$$

(2) VBE step: take the functional derivative of $\mathrm{F}\left[q_{\boldsymbol{\Theta}}(\boldsymbol{\theta}), q_{\boldsymbol{X}_{1: N+1}}\left(\boldsymbol{x}_{1: N+1}\right)\right]$ with respect to $q_{\boldsymbol{X}_{1: N+1}}\left(\boldsymbol{x}_{1: N+1}\right)$ and set it equal to zero, i.e.

$$
\begin{aligned}
& \frac{\partial \mathrm{F}\left[q_{\boldsymbol{\Theta}}(\boldsymbol{\theta}), q_{\boldsymbol{X}_{1: N+1}}\left(\boldsymbol{x}_{1: N+1}\right)\right]}{\partial q_{\boldsymbol{X}_{1: N+1}}\left(\boldsymbol{x}_{1: N+1}\right)} \\
& =-\ln q_{\boldsymbol{X}_{1: N+1}}\left(\boldsymbol{x}_{1: N+1}\right)+\ln \pi_{\boldsymbol{X}_{1}}\left(\boldsymbol{x}_{1}\right)-1+\sum_{k=1}^{N} \iint q_{\Gamma \Sigma}(\boldsymbol{\gamma}, \boldsymbol{\sigma}) \ln p_{\boldsymbol{Z}_{k} \mid \boldsymbol{\Gamma} \Sigma \boldsymbol{X}_{k}}\left(\boldsymbol{z}_{k} \mid \boldsymbol{\gamma}, \boldsymbol{\sigma}, \boldsymbol{x}_{k}\right) d \boldsymbol{\gamma} d \boldsymbol{\sigma}=0
\end{aligned}
$$

Then, we have

$$
q_{\boldsymbol{X}_{1: N+1}^{(t+1)}}\left(\boldsymbol{x}_{1: N+1}\right) \propto \exp \left\{-\frac{1}{2}\left[\left(\boldsymbol{x}_{1}-\boldsymbol{\mu}_{1}^{(t)}\right)^{\mathrm{T}} \boldsymbol{P}_{1}^{(t)^{-1}}\left(\boldsymbol{x}_{1}-\boldsymbol{\mu}_{1}^{(t)}\right)+\sum_{k=1}^{N} \mathrm{E}_{\boldsymbol{\Gamma} \boldsymbol{\Sigma}}\left[\left(\mathbf{z}_{k}-\boldsymbol{\gamma} \boldsymbol{x}_{k}\right)^{\mathrm{T}} \boldsymbol{\sigma}^{-1}\left(\mathbf{z}_{k}-\boldsymbol{\gamma} \boldsymbol{x}_{k}\right)\right]\right]\right\}
$$

For the expectation term, we have

$$
\begin{aligned}
\mathrm{E}_{\boldsymbol{\Gamma} \Sigma}\left[\left(\boldsymbol{z}_{k}-\boldsymbol{\gamma} \boldsymbol{x}_{k}\right)^{\mathrm{T}} \boldsymbol{\sigma}^{-1}\left(\boldsymbol{z}_{k}-\boldsymbol{\gamma} \boldsymbol{x}_{k}\right)\right] & =d\left(\boldsymbol{z}_{k}-\boldsymbol{\mu}_{\boldsymbol{\Gamma}}^{(t)} \boldsymbol{x}_{k}\right)^{\mathrm{T}} \boldsymbol{D}^{(t)^{-1}}\left(\boldsymbol{z}_{k}-\boldsymbol{\mu}_{\boldsymbol{\Gamma}}^{(t)} \boldsymbol{x}_{k}\right) \\
& +\boldsymbol{x}_{k}^{\mathrm{T}} \mathrm{E}_{\boldsymbol{\Gamma} \boldsymbol{\Sigma}}\left[\left(\boldsymbol{\gamma}-\boldsymbol{\mu}_{\gamma}^{(t)}\right)^{\mathrm{T}} \boldsymbol{\sigma}^{-1}\left(\boldsymbol{\gamma}-\boldsymbol{\mu}_{\gamma}^{(t)}\right)\right] \boldsymbol{x}_{k}
\end{aligned}
$$

The $(i, j)$ element of the expectation term in the above equation is

$$
\begin{aligned}
\mathrm{E}_{\boldsymbol{\Gamma} \boldsymbol{\Sigma}}\left[\left(\boldsymbol{\gamma}_{i}-\boldsymbol{\mu}_{\boldsymbol{\Gamma}, i}^{(t)}\right)^{\mathrm{T}} \boldsymbol{\sigma}^{-1}\left(\boldsymbol{\gamma}_{j}-\boldsymbol{\mu}_{\boldsymbol{\Gamma}, j}^{(t)}\right)\right] & =\operatorname{tr}\left\{\mathrm{E}_{\boldsymbol{\Gamma} \boldsymbol{\Sigma}}\left[\left(\boldsymbol{\gamma}_{j}-\boldsymbol{\mu}_{\boldsymbol{\Gamma}, j}^{(t)}\right)\left(\boldsymbol{\gamma}_{i}-\boldsymbol{\mu}_{\boldsymbol{\Gamma}, i}^{(t)}\right)^{\mathrm{T}} \boldsymbol{\sigma}^{-1}\right]\right\}=\operatorname{tr}\left[\left(\boldsymbol{\Pi}_{j i}^{(t)} \boldsymbol{\sigma}\right) \boldsymbol{\sigma}^{-1}\right] \\
& =\left(N_{s}+N_{o}\right) \boldsymbol{\Pi}_{j i}^{(t)}
\end{aligned}
$$

Then,

$$
\mathrm{E}_{\boldsymbol{\Gamma} \Sigma}\left[\left(\boldsymbol{\gamma}-\boldsymbol{\mu}_{\gamma}^{(t)}\right)^{\mathrm{T}} \boldsymbol{\sigma}^{-1}\left(\boldsymbol{\gamma}-\boldsymbol{\mu}_{\gamma}^{(t)}\right)\right]=\left(N_{s}+N_{o}\right) \boldsymbol{\Pi}^{(t)}
$$

Substituting Eqns. (B.4) and (B.6) into Eqn. (B.3) yields Eqn. (19). 
(3) VBM step: since a conjugate prior is used for $\boldsymbol{\Theta}$, posterior distributions have the same form and we only need to update the distribution parameters. Taking the functional derivative of F $\left[q_{\boldsymbol{\Theta}}(\boldsymbol{\theta}), q_{\boldsymbol{X}_{1: N+1}}\left(\boldsymbol{x}_{1: N+1}\right)\right]$ with respect to $q_{\Gamma \Sigma}(\gamma, \sigma)$ yields

$\frac{\partial \mathrm{F}\left[q_{\boldsymbol{\theta}}(\boldsymbol{\theta}), q_{X_{1: N+1}}\left(\boldsymbol{x}_{1: N+1}\right)\right]}{\partial q_{\Gamma \Sigma}(\gamma, \boldsymbol{\sigma})}=-\ln \frac{q_{\Gamma \Sigma}(\gamma, \boldsymbol{\sigma})}{\pi_{\Gamma \Sigma}(\gamma, \boldsymbol{\sigma})}-1+\sum_{k=1}^{N} \iint q_{\boldsymbol{X}_{k: k+1}}\left(\boldsymbol{x}_{k: k+1}\right) \ln p_{\boldsymbol{Z}_{k} \mid \boldsymbol{\Gamma} \boldsymbol{\Sigma} \boldsymbol{X}_{k}}\left(\boldsymbol{z}_{k} \mid \boldsymbol{\gamma}, \boldsymbol{\sigma}, \boldsymbol{x}_{k}\right) d \boldsymbol{x}_{k: k+1}$

Setting Eqn. (B.7) to zero gives

$q_{\boldsymbol{\Gamma} \boldsymbol{\Sigma}}^{(t+1)}(\boldsymbol{\gamma}, \boldsymbol{\sigma}) \propto \pi_{\boldsymbol{\Gamma} \boldsymbol{\Sigma}}(\boldsymbol{\gamma}, \boldsymbol{\sigma}) \exp \left[\sum_{k=1}^{N} \iint q_{\boldsymbol{X}_{k: k+1}}\left(\boldsymbol{x}_{k: k+1}\right) \ln p_{\boldsymbol{Z}_{k} \mid \boldsymbol{\Gamma} \boldsymbol{\Sigma} \boldsymbol{X}_{k}}\left(\boldsymbol{z}_{k} \mid \boldsymbol{\gamma}, \boldsymbol{\sigma}, \boldsymbol{x}_{k}\right) d \boldsymbol{x}_{k: k+1}\right]$

$\propto|\boldsymbol{\sigma}|^{-\frac{d+N+N_{S}+2 N_{o}+1}{2}} \exp \left\{-\frac{1}{2} \operatorname{tr}\left[\boldsymbol{D} \boldsymbol{\sigma}^{-1}+\boldsymbol{\Pi}^{-1}\left(\boldsymbol{\gamma}-\boldsymbol{\mu}_{\boldsymbol{\Gamma}}\right)^{\mathrm{T}} \boldsymbol{\sigma}^{-1}\left(\boldsymbol{\gamma}-\boldsymbol{\mu}_{\boldsymbol{\Gamma}}\right)+\mathrm{E}_{\boldsymbol{X}_{k: k+1}}\left[\left(\boldsymbol{z}_{k}-\boldsymbol{\gamma} \boldsymbol{x}_{k}\right)\left(\boldsymbol{z}_{k}-\boldsymbol{\gamma} \boldsymbol{x}_{k}\right)^{\mathrm{T}} \boldsymbol{\sigma}^{-1}\right]\right]\right\}$

$\propto|\boldsymbol{\sigma}|^{-\frac{\widehat{d}^{(t+1)}+N_{S}+2 N_{O}+1}{2}} \exp \left\{-\frac{1}{2} \operatorname{tr}\left[\boldsymbol{D}^{(t+1)} \boldsymbol{\sigma}^{-1}+\boldsymbol{\Pi}^{(t+1)^{-1}}\left(\boldsymbol{\gamma}-\boldsymbol{\mu}_{\boldsymbol{\Gamma}}^{(t+1)}\right)^{\mathrm{T}} \boldsymbol{\sigma}^{-1}\left(\boldsymbol{\gamma}-\boldsymbol{\mu}_{\boldsymbol{\Gamma}}^{(t+1)}\right)\right]\right\}$

in which $\boldsymbol{\Pi}^{(t+1)}, \boldsymbol{\mu}_{\boldsymbol{\Gamma}}^{(t+1)}, d^{(t+1)}$ and $\boldsymbol{D}^{(t+1)}$ are as specified following Eqn. (20).

(4) VBP step: similar to the above two steps, by taking the functional derivatives of

$\mathrm{F}\left[q_{\boldsymbol{\Theta}}(\boldsymbol{\theta}), q_{\boldsymbol{X}_{1: N+1}}\left(\boldsymbol{x}_{1: N+1}\right)\right]$ with respect to the prior $\pi_{\boldsymbol{X}_{1}}\left(\boldsymbol{x}_{1}\right)$, it is easy to verify $\pi_{\boldsymbol{X}_{1}}^{(t+1)}\left(\boldsymbol{x}_{1}\right)=q_{\boldsymbol{X}_{1}}^{(t+1)}\left(\boldsymbol{x}_{1}\right)$.

Thus, Eqn. (21) holds.

Since all the distributions involved belong to the exponential family, the VB-EMP estimation is statistically consistent and monotonically convergent given an initial point close to the true value [25]. Furthermore, following Fujimoto et al. [22], we can show the proposed algorithm is invariant under coordinate transformations.

Proof of Lemma 5.1.

After the VBM step, we have from Eqn. (B.7)

$$
\ln \frac{q_{\Gamma \Sigma}(\gamma, \boldsymbol{\sigma})}{\pi_{\Gamma \Sigma}(\gamma, \boldsymbol{\sigma})}=\sum_{k=1}^{N} \iint q_{\boldsymbol{X}_{k: k+1}}\left(\boldsymbol{x}_{k: k+1}\right) \ln p_{\boldsymbol{Z}_{k} \mid \boldsymbol{\Gamma} \boldsymbol{\Sigma} \boldsymbol{X}_{k}}\left(\mathbf{z}_{k} \mid \boldsymbol{\gamma}, \boldsymbol{\sigma}, \boldsymbol{x}_{k}\right) d \boldsymbol{x}_{k: k+1}-1
$$

Substituting Eqn. (B.9) into Eqn. (B.1) gives

$$
\mathrm{F}\left[q_{\boldsymbol{\Theta}}(\boldsymbol{\theta}), q_{\boldsymbol{X}_{1: N+1}}\left(\boldsymbol{x}_{1: N+1}\right)\right]=-\int q_{\boldsymbol{X}_{1: N+1}}\left(\boldsymbol{x}_{1: N+1}\right) \ln \frac{q_{X_{1: N+1}}\left(x_{1: N+1}\right)}{\pi_{X_{1}}\left(x_{1}\right)} d x_{1: N+1}+1
$$

Using Eqn. (19) and the notations from Lemma 4.1, we have

$$
\begin{aligned}
& q_{\boldsymbol{X}_{1: N+1}}^{(t+1)}\left(\boldsymbol{x}_{1: N+1}\right) \propto|\widetilde{\boldsymbol{Q}}|^{\frac{N}{2}}|\widetilde{\boldsymbol{R}}|^{\frac{N}{2}} \exp \left\{-\frac{1}{2}\left[\left(\boldsymbol{x}_{1}-\boldsymbol{\mu}_{1}^{(t)}\right)^{\mathrm{T}} \boldsymbol{P}_{1}^{(t)^{-1}}\left(\boldsymbol{x}_{1}-\boldsymbol{\mu}_{1}^{(t)}\right)\right.\right. \\
&\left.\left.+\sum_{k=1}^{N}\left(\left[\begin{array}{c}
\boldsymbol{x}_{k+1} \\
\widetilde{\boldsymbol{y}}_{k}
\end{array}\right]-\left[\begin{array}{c}
\widetilde{\boldsymbol{A}} \\
\widetilde{\boldsymbol{C}}
\end{array}\right] \boldsymbol{x}_{k}\right)^{\mathrm{T}}\left[\begin{array}{cc}
\widetilde{\boldsymbol{Q}} & \mathbf{0}_{N_{S} \times\left(N_{s}+N_{o}\right)} \\
\mathbf{0}_{\left(N_{S}+N_{o}\right) \times N_{s}} & \widetilde{\boldsymbol{R}}
\end{array}\right]^{-1}\left(\left[\begin{array}{c}
\boldsymbol{x}_{k+1} \\
\widetilde{\boldsymbol{y}}_{k}
\end{array}\right]-\left[\begin{array}{c}
\widetilde{\boldsymbol{A}} \\
\widetilde{\boldsymbol{C}}
\end{array}\right] \boldsymbol{x}_{k}\right)\right]\right\}
\end{aligned}
$$

Inserting Eqn. (B.11) into Eqn. (B.10) results in Eqn. (36). This completes the proof. 


\section{Appendix C: Partial derivatives of eigenvalue and eigenvector}

In this appendix we show how the terms in the Taylor series expansion in Eqn. (43) can be efficiently computed. It is a modification of the algorithm originally proposed by Vlach and Singhal [37].

For generality, we consider the partial derivatives of eigenvalue $\lambda$ and eigenvector $\psi$ with respect to the parameter $\theta$ in generalized eigenvalue problem

$$
\boldsymbol{A}(\theta) \boldsymbol{\psi}=\lambda \boldsymbol{B}(\theta) \boldsymbol{\psi}
$$

where $\boldsymbol{A}(\theta) \in \mathbb{R}^{n \times n}$ and $\boldsymbol{B}(\theta) \in \mathbb{R}^{n \times n}$. For simplicity, we will ignore the argument $\theta$ in $\boldsymbol{A}$ and $\boldsymbol{B}$ in the following derivations. If we set $\boldsymbol{B}=\boldsymbol{I}_{n}$, it automatically degenerates to an eigenvalue problem. Rewrite Eqn. (C.1) into an equivalent form

$$
H \boldsymbol{\psi}=(\boldsymbol{A}-\lambda \boldsymbol{B}) \boldsymbol{\psi}=\mathbf{0}
$$

where $\boldsymbol{H}=\boldsymbol{A}-\lambda \boldsymbol{B}$. Consider the LU decomposition of $\boldsymbol{H}$,

$$
\boldsymbol{P H}=\boldsymbol{L U}
$$

where $\boldsymbol{P}$ is a permutation matrix, $\boldsymbol{L}$ is a lower triangular matrix with unit entries along the diagonal and $\boldsymbol{U}$ is an upper triangular matrix. Note that $\boldsymbol{L}$ and $\boldsymbol{U}$ are both complex if $\lambda$ is a complex eigenvalue. Use the property of matrix determinant,

$$
\operatorname{det}(\boldsymbol{H})=\operatorname{det}(\boldsymbol{L}) \operatorname{det}(\boldsymbol{U})=\operatorname{det}(\boldsymbol{U})
$$

where we have used the fact that $\operatorname{det}(\boldsymbol{L})=1$. If $\lambda$ satisfies Eqn. (C.1), $\operatorname{det}(\boldsymbol{H})=0$, and $\operatorname{thus} \operatorname{det}(\boldsymbol{U})=0$, meaning that the matrix $\boldsymbol{U}$ must has a zero element along its diagonal. Assuming that this zero element is in the last column, i.e. $u_{n n}=0$, we obtain the following relation

$$
\frac{\mathrm{d} u_{n n}}{\mathrm{~d} \theta}=\frac{\partial u_{n n}}{\partial \lambda} \frac{\partial \lambda}{\partial \theta}+\frac{\partial u_{n n}}{\partial \theta}=0
$$

To find the terms $\partial u_{n n} / \partial \lambda$ and $\partial u_{n n} / \partial \theta$, we differentiate Eqn. (C.3) by an arbitrary parameter $h$

$$
\boldsymbol{P} \frac{\partial \boldsymbol{H}}{\partial h}=\frac{\partial \boldsymbol{L}}{\partial h} \boldsymbol{U}+\boldsymbol{L} \frac{\partial \boldsymbol{U}}{\partial h}
$$

Define two vectors $\boldsymbol{x}$ and $\boldsymbol{y}$ as follows:

$$
\begin{aligned}
& \boldsymbol{L}^{*} \boldsymbol{x}=\boldsymbol{e}_{n} \\
& \boldsymbol{U} \boldsymbol{y}=\mathbf{0}
\end{aligned}
$$

where $\boldsymbol{e}_{n}$ is the $n$-th column of the identity matrix and $*$ means the conjugate transpose. Since $\boldsymbol{y}$ can be arbitrarily normalized, we force its last element to be one, i.e. $y_{n}=1$.

Pre- and post-multiplying Eqn. (C.6) by $\boldsymbol{x}^{*}$ and $\boldsymbol{y}$ yields

$$
\boldsymbol{x}^{*} \boldsymbol{P} \frac{\partial \boldsymbol{H}}{\partial h} \boldsymbol{y}=\boldsymbol{x}^{*} \frac{\partial \boldsymbol{L}}{\partial h} \boldsymbol{U} \boldsymbol{y}+\boldsymbol{x}^{*} \boldsymbol{L} \frac{\partial \boldsymbol{U}}{\partial h} \boldsymbol{y}=\frac{\partial u_{n n}}{\partial h}
$$

By setting $h$ equal to $\lambda$ and $\theta$, we can obtain $\partial u_{n n} / \partial \lambda$ and $\partial u_{n n} / \partial \theta$, and $\partial \lambda / \partial \theta$ as a consequence. 
Now, let us consider the partial derivative of eigenvectors. Starting with Eqn. (C.2), we can get the partial derivatives on both side using chain rule:

$$
\left(\frac{\partial H}{\partial \theta}+\frac{\partial H}{\partial \lambda} \frac{\partial \lambda}{\partial \theta}\right) \boldsymbol{\psi}+\boldsymbol{H} \frac{\partial \psi}{\partial \theta}=\mathbf{0}
$$

Rearranging the above equation and inserting Eqn. (C.3) gives

$$
\boldsymbol{U} \frac{\partial \psi}{\partial \theta}=-\boldsymbol{L}^{-1} \boldsymbol{P}\left(\frac{\partial H}{\partial \theta}+\frac{\partial H}{\partial \lambda} \frac{\partial \lambda}{\partial \theta}\right) \boldsymbol{\psi}
$$

Eqn. (C.10) is linear equation with all terms on the right hand side are known from previous steps. However, the matrix $\boldsymbol{U}$ is singular, so we must enforce one additional constraint. Since eigenvectors are only determined within a constant factor, they are often normalized such that the magnitude of their linear transformation is equal to one, i.e.

$$
(\boldsymbol{T} \boldsymbol{\psi})^{*}(\boldsymbol{T} \boldsymbol{\psi})=1
$$

Differentiating this constraint gives

$$
(\boldsymbol{T} \boldsymbol{\psi})^{*} \boldsymbol{T} \frac{\partial \psi}{\partial \theta}=0
$$

Combining Eqns. (C.10) and (C.12) yields

$$
\left[\begin{array}{l}
\boldsymbol{U}_{1: n-1,:} \\
(\boldsymbol{T} \boldsymbol{\psi})^{*} \boldsymbol{T}
\end{array}\right] \frac{\partial \boldsymbol{\psi}}{\partial \theta}=-\boldsymbol{L}^{-1} \boldsymbol{P}\left(\frac{\partial \boldsymbol{H}}{\partial \theta}+\frac{\partial \boldsymbol{H}}{\partial \lambda} \frac{\partial \lambda}{\partial \theta}\right) \boldsymbol{\psi}
$$

where $\boldsymbol{U}_{1: n-1,:}$ means the first $n-1$ rows of matrix $\boldsymbol{U}$. Now, the matrix on the left hand side becomes square and full-rank, so that $\partial \boldsymbol{\psi} / \partial \theta$ can be uniquely determined.

Case 1: $\partial \lambda / \partial a_{i j}$ and $\partial \boldsymbol{\psi} / \partial a_{i j}$.

We have $\boldsymbol{H}=\boldsymbol{A}-\lambda \boldsymbol{I}_{N_{s}}$, then $\partial \boldsymbol{H} / \partial \lambda=-\boldsymbol{I}_{N_{S}}$ and $\partial \boldsymbol{H} / \partial a_{i j}=\boldsymbol{e}_{i} \boldsymbol{e}_{\boldsymbol{j}}^{\mathrm{T}}$.

$$
\frac{\partial u_{n n}}{\partial \lambda}=-\boldsymbol{x}^{*} \boldsymbol{P} \boldsymbol{y}, \frac{\partial u_{n n}}{\partial a_{i j}}=\boldsymbol{x}^{*} \boldsymbol{P} \boldsymbol{e}_{i} \boldsymbol{e}_{j}^{\mathrm{T}} \boldsymbol{y}=\left(\boldsymbol{x}^{*} \boldsymbol{P}\right)_{i} y_{j}
$$

According to Eqn. (C.5), we obtain

$$
\frac{\partial \lambda}{\partial a_{i j}}=\frac{\left(\boldsymbol{x}^{*} \boldsymbol{P}\right)_{i} y_{j}}{\boldsymbol{x}^{*} \boldsymbol{P} \boldsymbol{y}}
$$

Or we can write it into a matrix form as

$$
\frac{\partial \lambda}{\partial \operatorname{vec}(\boldsymbol{A})}=\frac{\boldsymbol{y}^{\mathrm{T}} \otimes\left(\boldsymbol{x}^{*} \boldsymbol{P}\right)}{\boldsymbol{x}^{*} \boldsymbol{P} \boldsymbol{y}}
$$

Setting $\boldsymbol{T}=\boldsymbol{I}_{N_{S}}$ and substituting known terms into Eqn. (C.13) gives

$$
\left[\begin{array}{c}
\boldsymbol{U}_{1: n-1,:} \\
\boldsymbol{\psi}^{*}
\end{array}\right] \frac{\partial \boldsymbol{\psi}}{\partial a_{i j}}=\boldsymbol{L}^{-1} \boldsymbol{P}\left(\frac{\partial \lambda}{\partial a_{i j}} \boldsymbol{I}_{N_{S}}-\boldsymbol{e}_{i} \boldsymbol{e}_{j}^{\mathrm{T}}\right) \boldsymbol{\psi}
$$


Or we can write it into a matrix form as

$$
\left[\begin{array}{c}
\boldsymbol{U}_{1: n-1,:} \\
\boldsymbol{\psi}^{*}
\end{array}\right] \frac{\partial \boldsymbol{\psi}}{\partial \operatorname{vec}(\mathrm{A})}=\boldsymbol{L}^{-1} \boldsymbol{P}\left(\frac{\partial \lambda}{\partial \operatorname{vec}(\boldsymbol{A})} \otimes \boldsymbol{\psi}-\boldsymbol{\psi}^{\mathrm{T}} \otimes \boldsymbol{I}_{N_{S}}\right)
$$

Case 2: $\partial \lambda_{m i n} / \partial a_{m n}, \partial \mathbf{z} / \partial a_{m n}$ and $\partial \lambda_{m i n} / \partial c_{m n}, \partial \mathbf{z} / \partial c_{m n}$

We have $\boldsymbol{H}=\boldsymbol{P}_{1} \boldsymbol{\varphi}_{i}^{\mathrm{T}} \boldsymbol{C}^{\mathrm{T}} \boldsymbol{C} \boldsymbol{\varphi}_{i} \boldsymbol{P}_{1}-\lambda_{\min } \boldsymbol{P}_{2} \boldsymbol{\varphi}_{i}^{\mathrm{T}} \boldsymbol{C}^{\mathrm{T}} \boldsymbol{C} \boldsymbol{\varphi}_{i} \boldsymbol{P}_{2}$, then $\partial \boldsymbol{H} / \partial \lambda_{\min }=-\boldsymbol{P}_{2} \boldsymbol{\varphi}_{i}^{\mathrm{T}} \boldsymbol{C}^{\mathrm{T}} \boldsymbol{C} \boldsymbol{\varphi}_{i} \boldsymbol{P}_{2}$ and

$$
\frac{\partial \boldsymbol{H}}{\partial a_{m n}}=\boldsymbol{P}_{1} \frac{\partial \boldsymbol{\varphi}_{i}{ }^{\mathrm{T}}}{\partial a_{m n}} \boldsymbol{C}^{\mathrm{T}} \boldsymbol{C} \boldsymbol{\varphi}_{i} \boldsymbol{P}_{1}+\boldsymbol{P}_{1} \boldsymbol{\varphi}_{i}^{\mathrm{T}} \boldsymbol{C}^{\mathrm{T}} \boldsymbol{C} \frac{\partial \boldsymbol{\varphi}_{i}}{\partial a_{m n}} \boldsymbol{P}_{1}-\lambda_{m i n}\left(\boldsymbol{P}_{2} \frac{\partial \boldsymbol{\varphi}_{i}{ }^{\mathrm{T}}}{\partial a_{m n}} \boldsymbol{C}^{\mathrm{T}} \boldsymbol{C} \boldsymbol{\varphi}_{i} \boldsymbol{P}_{2}+\boldsymbol{P}_{2} \boldsymbol{\varphi}_{i}^{\mathrm{T}} \boldsymbol{C}^{\mathrm{T}} \boldsymbol{C} \frac{\partial \boldsymbol{\varphi}_{i}}{\partial a_{m n}} \boldsymbol{P}_{2}\right)
$$

Thus, we have

$$
\frac{\partial u_{22}}{\partial \lambda_{\min }}=-\boldsymbol{x}^{*} \boldsymbol{P} \boldsymbol{P}_{2} \boldsymbol{\varphi}_{i}^{\mathrm{T}} \boldsymbol{C}^{\mathrm{T}} \boldsymbol{C} \boldsymbol{\varphi}_{i} \boldsymbol{P}_{2} \boldsymbol{y}, \frac{\partial u_{22}}{\partial a_{m n}}=\boldsymbol{x}^{*} \boldsymbol{P} \frac{\partial \boldsymbol{H}}{\partial a_{m n}} \boldsymbol{y}, \frac{\partial \lambda_{m i n}}{\partial a_{m n}}=\boldsymbol{x}^{*} \boldsymbol{P} \frac{\partial \boldsymbol{H}}{\partial a_{m n}} \boldsymbol{y} /\left(\boldsymbol{x}^{*} \boldsymbol{P} \boldsymbol{P}_{2} \boldsymbol{\varphi}_{i}^{\mathrm{T}} \boldsymbol{C}^{\mathrm{T}} \boldsymbol{C} \boldsymbol{\varphi}_{i} \boldsymbol{P}_{2} \boldsymbol{y}\right)
$$

For the sensitivity of eigenvector,

$$
\left[\begin{array}{l}
\boldsymbol{U}_{1,:}^{\mathrm{T}} \\
\boldsymbol{z}^{\mathrm{T}} \boldsymbol{P}_{2} \boldsymbol{\varphi}_{i}^{\mathrm{T}} \boldsymbol{C}^{\mathrm{T}} \boldsymbol{C} \boldsymbol{\varphi}_{i} \boldsymbol{P}_{2}
\end{array}\right] \frac{\partial \boldsymbol{z}}{\partial a_{m n}}=\boldsymbol{L}^{-1} \boldsymbol{P}\left(\frac{\partial \lambda_{m i n}}{\partial a_{m n}} \boldsymbol{P}_{2} \boldsymbol{\varphi}_{i}^{\mathrm{T}} \boldsymbol{C}^{\mathrm{T}} \boldsymbol{C} \boldsymbol{\varphi}_{i} \boldsymbol{P}_{2}-\frac{\partial \boldsymbol{H}}{\partial a_{m n}}\right) \boldsymbol{z}
$$

Similarly,

$$
\frac{\partial \boldsymbol{H}}{\partial c_{m n}}=\boldsymbol{P}_{1} \boldsymbol{\varphi}_{i}^{\mathrm{T}} \boldsymbol{e}_{n} \boldsymbol{e}_{m}^{\mathrm{T}} \boldsymbol{C} \boldsymbol{\varphi}_{i} \boldsymbol{P}_{1}+\boldsymbol{P}_{1} \boldsymbol{\varphi}_{i}^{\mathrm{T}} \boldsymbol{C}^{\mathrm{T}} \boldsymbol{e}_{m} \boldsymbol{e}_{n}^{\mathrm{T}} \boldsymbol{\varphi}_{i} \boldsymbol{P}_{1}-\lambda_{m i n}\left(\boldsymbol{P}_{2} \boldsymbol{\varphi}_{i}^{\mathrm{T}} \boldsymbol{e}_{n} \boldsymbol{e}_{m}^{\mathrm{T}} \boldsymbol{C} \boldsymbol{\varphi}_{i} \boldsymbol{P}_{2}+\boldsymbol{P}_{2} \boldsymbol{\varphi}_{i}^{\mathrm{T}} \boldsymbol{C}^{\mathrm{T}} \boldsymbol{e}_{m} \boldsymbol{e}_{n}^{\mathrm{T}} \boldsymbol{\varphi}_{i} \boldsymbol{P}_{2}\right)
$$

Therefore,

$$
\frac{\partial u_{22}}{\partial a_{m n}}=\boldsymbol{x}^{*} \boldsymbol{P} \frac{\partial \boldsymbol{H}}{\partial c_{m n}} \boldsymbol{y}, \frac{\partial \lambda_{\min }}{\partial c_{m n}}=\boldsymbol{x}^{*} \boldsymbol{P} \frac{\partial \boldsymbol{H}}{\partial c_{m n}} \boldsymbol{y} /\left(\boldsymbol{x}^{*} \boldsymbol{P} \boldsymbol{P}_{2} \boldsymbol{\varphi}_{i}^{\mathrm{T}} \boldsymbol{C}^{\mathrm{T}} \boldsymbol{C} \boldsymbol{\varphi}_{i} \boldsymbol{P}_{2} \boldsymbol{y}\right)
$$

For the sensitivity of eigenvector,

$$
\left[\begin{array}{c}
\boldsymbol{U}_{1,:}^{\mathrm{T}} \\
\boldsymbol{z}^{\mathrm{T}} \boldsymbol{P}_{2} \boldsymbol{\varphi}_{i}^{\mathrm{T}} \boldsymbol{C}^{\mathrm{T}} \boldsymbol{C} \boldsymbol{\varphi}_{i} \boldsymbol{P}_{2}
\end{array}\right] \frac{\partial \boldsymbol{z}}{\partial c_{m n}}=\boldsymbol{L}^{-1} \boldsymbol{P}\left(\frac{\partial \lambda_{\min }}{\partial c_{m n}} \boldsymbol{P}_{2} \boldsymbol{\varphi}_{i}^{\mathrm{T}} \boldsymbol{C}^{\mathrm{T}} \boldsymbol{C} \boldsymbol{\varphi}_{i} \boldsymbol{P}_{2}-\frac{\partial \boldsymbol{H}}{\partial c_{m n}}\right) \boldsymbol{z}
$$

